\title{
The distributional incidence of growth: a non-anonymous and rank dependent approach ${ }^{* \dagger}$
}

\author{
Flaviana Palmisano ${ }^{\ddagger}$ and Vito Peragine ${ }^{\S}$
}

\begin{abstract}
This paper provides a normative framework for the assessment of the distributional incidence of growth. By removing the anonymity axiom, such framework is able to evaluate the individual income changes over time and the reshuffling of individuals along the income distribution that are determined by the pattern of income growth. We adopt a rank dependent social welfare function expressed in terms of initial rank and individual income change and we obtain partial and complete dominance conditions over different growth paths. These dominance conditions account for the different components determining the overall impact of growth, that is the size of growth and its vertical and horizontal incidence. We then provide an empirical application for Italy: this analysis shows the distributional impact of the recent economic crisis suffered by the Italian populaltion.
\end{abstract}

Keywords: Growth; Pro-poor; Inequality; Income mobility; Dominance.

JEL classification: D31, D63, D71.

\section{Introduction}

In recent years a new literature has emerged, both theoretical and empirical, on the measurement of the distributive impact of growth (see Bourguignon, 2003, 2004; Ferreira, 2010; Son, 2004; Ravallion and Chen, 2003). Different tools have been proposed for the evaluation of the pro-poorness of growth (see Duclos, 2009; Kakwani and Son, 2008; Kraay, 2006; Kakwani and Pernia, 2000; Essama-Nssah, 2005; Essama-Nssah and Lambert, 2009; Zheng, 2010), and different decompositions have been obtained that relate the concepts of growth to poverty and inequality (see Datt and Ravallion; 1992; Jenkins and Van Kerm, 2006). One basic tool used in this literature is the growth incidence curve (GIC), measuring the quantile-specific rate of economic growth between two points in time as a function of each percentile (Ravallion and Chen, 2003).

In this literature the growth process is basically analyzed by comparing the pre-growth and post-growth distribution. There is a clear analogy here between the transformation of an income distribution throughout the effect of growth and the transformation obtained as the effect of an income tax. In the same way as progressive taxation reduces inequality, a "progressive" growth

\footnotetext{
${ }^{*}$ We are very grateful to Stephen Jenkins and Philippe Van Kerm for detailed and useful comments, to Francois Bourguignon, Dirk Van de gaer and Valentino Dardanoni for fruitful discussions and to people attending the fourth meeting of the Society for the Study of Economic Inequality (ECINEQ), July 2011 in Catania (Italy).

${ }^{\dagger}$ Paper presented to the fourth meeting of the Society for the Study of Economic Inequality (ECINEQ), 18-21 July 2011, Catania (Italy).

$\ddagger$ University of Bari. E-mail: flaviana.palmisano@gmail.com

$\S$ University of Bari. E-mail: v.peragine@dse.uniba.it
} 
reduces the inequality in a distribution. In fact, such analogy has been deeply explored, and different results in the progressivity literature have been applied to this context (see Bénabou and Ok, 2001).

Now, with very few recent exceptions that will be discussed below, all this literature, when analyzing the distributional impact of growth, basically compares the phenomena under scrutiny before and after the growth process has taken place. Hence, for instance, to measure the propoorness of growth, the poverty levels (measured, say, according to the headcount or the poverty gap indices) are computed in the two periods of time and then compared. The same for different distributional indices. If this procedure is all right when one is interested in measuring the pure distributional change that takes place, this is unsatisfactory if one is interested in evaluating growth in terms of social welfare: from this view point, it can make a big difference if the poor people in the first period are still the same poor people in the following period, thereby experiencing chronic poverty, or if there has been a substantial reshuffling of the individual positions in the population. To capture this aspect one needs to remove a basic assumption used in the literature: the axiom of anonymity. According to this axiom, distributional measures are required to be invariant to permutations of income vectors. As a consequence, the individual income dynamics along the distribution is ignored.

We believe, on the contrary, that, for the welfare evaluation of growth, the identities of individuals do matter. This information allows to find out who are the winners and the losers from growth and to focus on the individual income transformations that take place during the growth process, a useful information, for example, in the evaluation of the efficacy of policy reforms. This information is usually hidden by the anonymity assumption in the standard approach. We propose, instead, an analytical framework for the normative assessment and comparison of growth processes, within which the evaluation of a growth process depends on the evaluation of the income change experienced by each individual in that society.

Our approach is close in spirit to Grimm (2007), who formally develops the concept of nonanonymous GIC (na-GIC). The na-GIC measures the individual-specific rate of economic growth between two points in time, thus comparing the income of individuals which were in the same initial position, independently of the position they acquire in the final distribution of income. Bourguignon (2011) and Jenkins and Van Kerm (2011) also contribute to this strand of the literature by proposing a normative justification for the use of na-GIC in the evaluation of growth. Bourguignon (2011) develops complete and partial dominance conditions to rank growth processes taking place on the same base-initial distribution of income. For, he adopts a utilitarian social welfare function (SWF), sensitive to the horizontal and vertical inequality of growth. Jenkins and Van Kerm (2011) develop complete and partial dominance conditions to rank growth processes, taking place on different initial distributions of income. They adopt a rank dependent SWF, sensitive to the vertical impact of growth.

In this paper we consider bivariate distributions of initial incomes and income changes: we first identify individuals according to their rank in the initial distribution of income; then, we evaluate the income evolution of each of these individuals. We provide new partial and complete dominance conditions for ranking growth processes, taking place on different initial distributions, and show that these conditions can be interpreted in terms of non-anonymous Growth Incidence Curve. However, differently from Bourguignon (2011), we adopt a rank dependent approach to social welfare, and differently from Jenkins and Van Kerm (2011), we also focus on the horizontal inequality of growth. Finally we show the empirical relevance of our framework, by implementing it in order to analyze the distributional impact of growth in Italy in the last decade. A very limited growth has been characterizing this country since the beginning of the new millennium. In fact, the 
average household income increased by $2 \%$ for the period $2002-2004$ and by $2.6 \%$ for the $2004-2006$. These periods of limited growth were finally followed by a long spell of negative growth during the recent economic crisis. In particular, the equivalent disposable income of the Italian households decreased by $2.6 \%$ during the $2006-2008$ period and by $0.6 \%$ during the $2008-2010$ (Banca d'Italia, 2008, 2012).

It is therefore interesting to understand wether the non-anonymous perspective to the evaluation of growth can provide useful information to complement the actual knowledge on the economic crisis in Italy. For, we use the Bank of Italy "Survey on Household Income and Wealth" (SHIW) in order to assess the distributional impact of growth in Italy. In particular, we consider four of the most recent available waves to compare 2002-2006 growth episode against the 2006-2010. The overall result of our analysis suggests that the impact of the crisis has been severe according to different features of growth.

The work is structured as follows. In section 2 we outline the framework. In section 3 we present the theoretical results. In section 4 we provide the empirical illustration. In section 5 we conclude.

\section{The framework}

Let $\mathbf{y}_{t}$ be the distribution of income at time $t$, the initial period, with cumulative distribution function (cdf) $F\left(y_{t}\right)$ and let $\delta=y_{t+1}-y_{t}$ be the income growth experienced by each individual in moving from date $t$ to $t+1$. Our aim is to evaluate the distributional incidence of growth taking place over $\mathbf{y}_{t}$, according to the non-anonymous perspective. Therefore we need to keep track of the identity of individuals. We assume that such identity is represented by their membership to a specific quantile group at time $t$. We partition $F\left(y_{t}\right)$ into quantile groups and we identify each individual according to the initial quantile group they belong to. We index each subgroup by $i=1, \ldots, n$ in increasing order, starting from the poorest to the richest, and we denote by $q_{i}$ the share of individuals in quantile ${ }^{1}$ group $i$, therefore $i$ - the information about individuals' identity corresponds to their rank in $F\left(y_{t}\right)$. Hence, for each initial quantile group $i$ it is possible to observe a distribution of income change with ${ }^{2} \operatorname{cdf} F_{i}(\delta)$ and $\delta_{i}^{(t, t+1)}(p)$ representing the income change of those individuals ranked $p$ in $F_{i}(\delta)$. Thus, $\Delta_{i}^{(t, t+1)}(p)=\int_{0}^{p} \delta_{i}^{(t, t+1)}(q) d q / p$ is the average income growth for those at the bottom $p$ quantiles within that initial quantile group $3^{3}$ and $\Delta_{i}^{(t, t+1)}(1)$ the income growth of that initial quantile group. We define by $\Delta^{(t, t+1)} \mid y_{t}$ the income transformation process of all individuals conditional on their identity and by $D$ the set of admissible growth paths.

We are interested in ranking members of $D$ from a normative perspective and we assume that such ranking can be represented by a social evaluation function, $W: D \longrightarrow \mathbb{R}$. Given the assumption of non-anonymity, $W$ will depend not only on the extent of the individual growth but also on the

\footnotetext{
${ }^{1}$ Obviously, $q_{i}=q_{j} \forall i, j=1, \ldots n$.

${ }^{2}$ Letting $F_{i}(\delta)$ be the cdf of the individual income change within each initial quantile group, $p=F_{i}(\delta) \Longleftrightarrow$ $\delta_{i}(p)=F_{i}^{-1}(p), \delta_{i}(p):=\inf \left\{y: F_{i}(\delta) \geq p\right\} . \delta_{i}(p)$ is the left inverse function, denoting the level of income change of individuals ranked $p$ in $F_{i}(\delta), i=1, \ldots, n$.

${ }^{3}$ Our framework can be extended to consider proportionate growth. All the dominance results will hold for distributions of proportionate income change. But the relationship with the na-GIC will be different.

The adoption of an absolute income change finds its justification in the absolute approach to the pro-poorness of growth. According to this approach what matters is that poor individuals experience positive growth, independently of the level of growth experienced by rich individuals.
} 
individual identity. According to this idea, a social preference over growth processes can be defined by:

$$
W=\sum_{i=1}^{n} q_{i} \int_{0}^{1} v_{i}(p) \delta_{i}^{(t, t+1)}(p) d p
$$

Eq. (1) aggregates the income change experienced by each individual, giving different weight to different individual $4_{4}^{4}$ In particular, $\int_{0}^{1} v_{i}(p) \delta_{i}^{(t, t+1)}(p) d p$ is the social evaluation ${ }^{5}$ of the growth experienced by the individuals belonging to a given initial quantile group $i=1, \ldots, n$. It is expressed by a weighted average of the income changes of its members, where each income change is weighted according to the position of each member in $F_{i}(\delta)$. The function $v_{i}(p):[0,1] \longrightarrow \Re_{+}$expresses the weight attached by the society to the change in income experienced by individuals ranked $p$ in $F_{i}(\delta)$ and $v_{i}(p) \delta_{i}^{(t, t+1)}(p)$ is the contribution of these individuals to the social evaluation of growth. The overall social evaluation of growth is then obtained by applying an additional weighted aggregation. That is, we aggregate the social evaluation of growth corresponding to each initial quantile group, weighted by the relevant population share, using initial quantile dependent weights. In sum, $W$ expresses concerns for both the initial and final economic situation of the individuals it represents. The set of weight functions specifying the social evaluation of growth, $\left\langle v_{1}(p), \ldots, v_{n}(p)>\right.$, will be called "weight profile". Different preferences over growth processes are encompassed in this framework by selecting different classes of "social weight" profiles. It is clear form eq. (1) that the differences in the initial distribution of income are neutralized. This enables to evaluate growth processes that take place over different initial distributions of income 6 .

\subsection{Properties}

In this section we discuss the properties that $W$ must satisfy. Consequently, different families of $W$ are derived, depending of the restrictions imposed on the weight function.

The first property is a standard monotonicity assumption.

Axiom 0 (Pro-Growth) For all $i=1, \ldots, n$, for all $p \in[0,1]$

$$
v_{i}(p) \geq 0
$$

According to this axiom a positive income change generates an increment of $W$, or at most leaves the social evaluation of growth unaffected; a negative income growth causes a reduction, or at most

\footnotetext{
${ }^{4}$ This approach is based on the idea that a social planner cares about the initial economic condition of inidividuals. For example, the initial poverty status of individuals might hamper their potential future development.

There is a rooted consensus that early stages of life matter more than later life periods in shaping the individual's lifetime well-being. It can be argued that the more poverty a person suffers in his childhood, the less is the person's future well-being conditional on any given level of income. This impact is taken into account, in our framework, through relaxing anonymity and ordering distribution partitioning the population according to their belonging in the initial distribution of income.

${ }^{5}$ See Yaari (1988), Donaldson and Weymark (1980), Aaberge (2001). See also Zoli (2000) and Peragine (2002) for alternative applications.

${ }^{6}$ Note the main difference with Borguignon (2011). In fact, while he conditions income changes to initial income levels and requires information about the density distribution of the base-year initial income distribution, we do not require such information, since we condition income changes on initial rank. This implies that we can use our framework to compare growth processes that take place over different initial distributions of income.
} 
does not affect it. Let $\mathbf{V}=\left\{\left\langle v_{1}(p), \ldots, v_{n}(p)>\right.\right.$ : Axiom 0 holds $\}$ and let $\mathbf{W}$ be the class of $W$ based on the weight profile $\mathbf{V}$.

We proceed by imposing aversion to vertical inequality.

Axiom 1 (Pro-poor Growth) For all $p \in[0,1]$, for all $i=1, \ldots, n$

$$
v_{i}(p) \geq v_{i+1}(p)
$$

This is expression of the transfer-sensitivity principle in the context of income growth among individuals having different rank in $F\left(y_{t}\right)$. That is, a transfer of a small amount of income change from an individual ranked $p$ in a richer initial quantile group $i+1$ to an individual ranked $p$ in a poorer initial quantile group $i$ does not decrease $W$. According to Axiom 1 , the social evaluation of growth would increase more if the income change concerns the initially poorest individuals. Let $\mathbf{V}_{\mathbf{1}}=\left\{\left\langle v_{1}(p), \ldots, v_{n}(p)\right\rangle\right.$ : Axioms 0 and 1 hold $\}$ and let $\mathbf{W}_{1}$ be the class of $W$ based on the weight profile $\mathbf{V}_{1}$.

Axiom 2 (Diminishing Pro-poor Growth) For all $p \in[0,1]$, for all $i=1, \ldots, n$

$$
v_{i-1}(p)-v_{i}(p) \geq v_{i}(p)-v_{i+1}(p)
$$

This axiom states that the weight given to the income change of individuals, ranked the same in different initial quantile group distributions, decreases more the lower is the initial rank of those individuals. That is, the poorer is the initial quantile group to which an individual belongs, the more sensitive is $W$ to the progressivity of the growth process under scrutiny. Let $V_{\mathbf{1 2}}=\{<$ $v_{1}(p), \ldots, v_{n}(p)>$ : Axioms 0,1 and 2 hold $\}$ and let $\mathrm{W}_{12}$ be the class of $W$ constructed in (1) and based on the weights profile $V_{12}$.

Note that in all these axioms, no concern is expressed with respect to horizontal equity, that is how individuals with same initial economic conditions - belonging to the same initial quantile group - are affected by growth. The following axiom, instead, goes in this direction.

Axiom 3 (Horizontally Equal Gains) For all $p \in[0,1]$, for all $i=1, \ldots, n$

$$
v_{i}^{\prime}(p) \leq 0
$$

This axiom states that a social planner evaluates more a growth process under which individuals with same starting economic conditions get equal gains. This implies that $W$ will be higher the lower is the inequality in the incidence of growth for individuals belonging to the same initial quantile group. This kind of social evaluation function captures inequality in gains conditionally on initial rank. Let $V_{\mathbf{3}}=\left\{\left\langle v_{1}(p), \ldots, v_{n}(p)\right\rangle\right.$ : Axioms 0 and 3 hold $\}$ and let $\mathrm{W}_{3}$ be the class of $W$ based on the weight profile in $V_{3}$.

Axiom 3* (Pro-poor Horizontally Equal Gains) For all $p \in[0,1]$, for all $i=1, \ldots, n$

$$
v_{i}^{\prime}(p) \leq v_{i+1}^{\prime}(p) \leq 0
$$

This axiom encompasses the joint effect of being ranked differently in the initial distribution of income and experiencing a different incidence of growth in each $i$. The same progressive transfer of income change is evaluated differently if it takes place in different initial quantile groups. $W$ increases more, the lower is the initial quantile group, within which the progressive transfer of income change takes place. Thus, the lower is the level of inequality of the individual gains within the poorest initial quantile groups, the higher is $W$. Let $\mathbf{V}_{13^{*}}=\left\{\left\langle v_{1}(p), \ldots, v_{n}(p)\right\rangle\right.$ : Axioms 0,1 and $3^{*}$ hold $\}$ and let $\mathbf{W}_{13^{*}}$ be the class of $W$ based on the weight profile $\mathbf{V}_{13^{*}}$. 
Axiom 4 (Horizontal Inequality Neutrality in Gains) For all $p \in[0,1]$, for all $i=1, \ldots, n$, $\exists \beta_{i} \in \Re$ such that

$$
v_{i}(p)=\beta_{i}
$$

This axiom states that $W$ is neutral to the inequality in each $i$. Therefore, a social planner adopting this preference would give the same weight to the income change of individuals ranked the same in the initial distribution of income. Let $\mathbf{V}_{\mathbf{4}}=\left\{\left\langle v_{1}(p), \ldots, v_{n}(p)\right\rangle\right.$ : Axioms 0 and 4 hold $\}$ and let $\mathbf{W}_{4}$ be the class of $W$ based on the weight profile $\mathbf{V}_{4}$. Furthermore, let $\mathbf{V}_{\mathbf{1 4}}=\{<$ $v_{1}(p), \ldots, v_{n}(p)>$ : Axioms 0,1 and 4 hold $\}$ and let $\mathbf{W}_{14}$ be the class of $W$ based on the weight profile $\mathbf{V}_{14}$. Last, let $\mathbf{V}_{\mathbf{1 2 4}}=\left\{\left\langle v_{1}(p), \ldots, v_{n}(p)\right\rangle\right.$ : Axioms $0,1,2$ and 4$\}$ and let $\mathbf{W}_{124}$ be the class of $W$ based on the weight profile $\mathbf{V}_{124}$.

\section{Results}

In this section we discuss the dominance conditions corresponding to different classes of social welfare evaluation functions $W$, all the proofs are gathered in the theoretical Appendix.

We start considering the class of $W$ satisfying the pro-growth axiom 7

Proposition 1 For all $\Delta_{A}^{(t, t+1)} \mid y_{t}$ and $\Delta_{B}^{(t, t+1)} \mid y_{t} \in D, W_{A} \geq W_{B}, \forall W \in \mathbf{W}$ if and only if

$$
\delta_{A i}^{(t, t+1)}(p) \geq \delta_{B i}^{(t, t+1)}(p) \forall i=1, \ldots, n, \forall p \in[0,1]
$$

The condition expressed in eq. (2) is a first order dominance. Given two distributions of income change $F_{A i}(\delta)$ and $F_{B i}(\delta)$, which are specific for each initial quantile group, the inverse of $F_{A i}(\delta)$, that is $\delta_{A i}^{(t, t+1)}(p)$, must lie nowhere above the inverse of $F_{B i}(\delta)$, that is $\delta_{B i}^{(t, t+1)}(p)$, for each $i=1, \ldots, n$. When we only impose pro-growth, to determine which growth process is socially preferable one needs to check, for every initial quantile group, that each individual in that group shows higher income growth in the dominating process than in the dominated one. This class of $W$ is expression of a simple efficiency-based criterion, no concern is expressed in terms of redistributional effect of growth. Therefore, the comparisons of growth processes according to this condition only rest on the extent of growth and it is independent from any redistributional features of growth.

We now turn to the family of $W \in W_{1}$.

Proposition 2 For all $\Delta_{A}^{(t, t+1)} \mid y_{t}$ and $\Delta_{B}^{(t, t+1)} \mid y_{t} \in D, W_{A} \geq W_{B}, \forall W \in \mathbf{W}_{1}$ if and only if

$$
\sum_{i=1}^{k} \delta_{A i}^{(t, t+1)}(p) \geq \sum_{i=1}^{k} \delta_{B i}^{(t, t+1)}(p), \forall k=1, \ldots, n, \forall p \in[0,1]
$$

The condition expressed in eq. (3) is a first order sequential distributional test, to be checked on the initial quantile group specific distribution of income change, starting from the lowest initial quantile group, then adding the second, then the third, and so on. The condition to be satisfied at each stage is a standard first order dominance of $\delta_{A i}^{(t, t+1)}(p)$ over $\delta_{B i}^{(t, t+1)}(p)$. That is, the quantile function of $F_{A i}(\delta), \delta_{A i}(p)$, must be higher than the corresponding one in $F_{B i}(\delta)$, and this

\footnotetext{
${ }^{7}$ Since we partition the initial distribution of income into quantile groups, by definition $q_{i}=q_{j}$ for all $i, j=1, \ldots n$ and they also will be the same across different distributions; therefore, the ordering provided by the dominance conditions we characterize here do not depend on $q_{i}$. This observation allows to leave out them in the derivation of our results.
} 
dominance must hold for every $p$, by sequential aggregation of the initial quantile groups. Hence, first we have to check that, for the poorest initial quantile group, $i=1$, at every $p$, the dominant distribution shows higher income changes than the dominated one. Then, we have to add the second lowest initial quantile group, and so on, and perform the same check at every ster 8 . In order to compare two processes according to this proposition, it is not enough to focus on the extent of growth; the final judgement will also depend on its vertical distributional impact.

The next family of $W$ we consider satisfies also axiom 2 .

Proposition 3 For all $\Delta_{A}^{(t, t+1)}\left|y_{t}, \Delta_{B}^{(t, t+1)}\right| y_{t} \in D, W_{A} \geq W_{B}, \forall W \in \mathbf{W}_{12}$ if and only if

$$
\sum_{i=1}^{j} \sum_{k=1}^{i} \delta_{F k}^{(t, t+1)}(p) \geq \sum_{i=1}^{j} \sum_{k=1}^{i} \delta_{G k}^{(t, t+1)}(p), \forall j=1, \ldots, n, \forall p \in[0,1]
$$

The condition expressed in eq. (4) is a first order "sequentially cumulated" distributional test. It provides a weaker dominance condition to be applied when it is not possible to rank distributions according to proposition 1 and 2 .

We now introduce a concern toward the horizontal incidence of growth, thus we turn to the family of $W \in W_{3}$.

Proposition 4 For all $\Delta_{A}^{(t, t+1)}\left|y_{t}, \Delta_{B}^{(t, t+1)}\right| y_{t} \in D, W_{A} \geq W_{B}, \forall W \in \mathbf{W}_{3}$ if and only if

$$
\int_{0}^{p} \delta_{A i}^{(t, t+1)}(q)-\delta_{B i}^{(t, t+1)}(q) d q \geq 0, \forall p \in[0,1], \forall i=1, \ldots, n
$$

The condition in eq. (5) is a second order distributional test to be applied at each initial quantile group. That is, for every $i=1, \ldots, n$, we have to check that the the average income growth for those at the bottom $p$ quantiles, within that initial quantile group, is higher in the dominating process than in the dominated one. If this condition is satisfied, it means that under the dominating process the gains (or losses) from growth are distributed more equally within each initial quantile groups than under the dominated one. Clearly, this evaluation is only concerned with the impact of growth among individuals with same initial economic status and it is independent from pro-poorness features.

Moving to the family of $W$ satisfying axiom $3^{*}$, we get the next result.

Proposition 5 For all $\Delta_{A}^{(t, t+1)}\left|y_{t}, \Delta_{A}^{(t, t+1)}\right| y_{t} \in D, W_{A} \geq W_{B}, \forall W \in \mathbf{W}_{13^{*}}$ if and only if

$$
\sum_{j=1}^{i} \int_{0}^{p} \delta_{A j}^{(t, t+1)}(q) d q \geq \sum_{j=1}^{i} \int_{0}^{p} \delta_{B j}^{(t, t+1)}(q) d q, \forall i=1, \ldots, n, \forall p \in[0,1]
$$

The condition expressed in eq. (6) is a second order sequential distributional test, to be checked starting from the poorest initial quantile group, then adding the second, then the third, and so on. The condition to be satisfied at each stage is that the cumulated sum of the individual income growth, within each initial quantile group, be higher in $\Delta_{A}^{(t, t+1)} \mid y_{t}$ than in $\Delta_{B}^{(t, t+1)} \mid y_{t}$. Hence, first we have to check that, for the poorest initial quantile, the cumulated income change of the $p$ least growing individuals in $F_{A i}(\delta)$ is higher than the corresponding one in $F_{B i}(\delta)$, and this dominance

\footnotetext{
${ }^{8}$ Recall that the sequential aggregation is to be implemented on individuals ranked the same in the different quantile groups being aggregated.
} 
must hold for all $p$. Then, we have to add the second lowest initial quantile, and so on, and perform the same check at every step. When the condition in this proposition is used to compare two growth processes, the evaluation becomes finer as it build upon all the possible features of growth, that is, the extent of growth and its vertical and horizontal incidence.

In is interesting to note that, in the special case in which each initial quantile group corresponds to a single individual, the results in eq. (2) and (5) are equivalent to a first order dominance of distributions of income change of individuals ranked according to their position in the initial distribution of income; whereas eq. (3) and (6) are equivalent to second order dominance conditions.

The next family of $W$ we consider satisfies horizontal inequality neutrality in gains.

Proposition 6 For all $\Delta_{A}^{(t, t+1)}\left|y_{t}, \Delta_{B}^{(t, t+1)}\right| y_{t} \in D, W_{A} \geq W_{B}, \forall W \in \mathbf{W}_{4}$ if and only if

$$
\Delta_{F i}^{(t, t+1)}(1) \geq \Delta_{G i}^{t, t+1}(1), \forall i=1, \ldots, n
$$

Eq. (7) is a first order direct dominance condition, or rank dominance, to be applied on distributions of income change means $9^{9}$ That is, we have to check that each initial quantile group shows higher mean income growth in $\Delta_{A}^{(t, t+1)} \mid y_{t}$ than in $\Delta_{B}^{(t, t+1)} \mid y_{t}$. Clearly this condition depends on the extent of growth of each initial quantile group and it is independent from distributional considerations.

We then consider the family of $W$ satisfying also axiom 1.

Proposition 7 For all $\Delta_{A}^{(t, t+1)}\left|y_{t}, \Delta_{B}^{(t, t+1)}\right| y_{t} \in D, W_{A} \geq W_{B}, \forall W \in \mathbf{W}_{14}$ if and only if

$$
\sum_{i=1}^{k} \Delta_{F i}^{(t, t+1)}(1) \geq \sum_{i=1}^{k} \Delta_{G i}^{(t, t+1)}(1), \forall k=1, \ldots, n
$$

Eq. (8) is a second order direct dominance to be applied on distributions of income change mean ${ }^{10}$ That is, we have to check that the cumulated sum of the initial quantile group specific mean income growth in higher in $\Delta_{A}^{(t, t+1)} \mid y_{t}$ than in $\Delta_{B}^{(t, t+1)} \mid y_{t}$. In this case, the dominance condition depends, not only on the extent of growth, but also on the incidence of growth on each initial quantile group; it is, instead, neutral with respect to the incidence of growth within each group.

Finally, we consider the family of $W \in W_{124}$.

Proposition 8 For all growth processes $\Delta_{A}^{(t, t+1)}\left|y_{t}, \Delta_{B}^{(t, t+1)}\right| y_{t} \in D, W_{A} \geq W_{B}, \forall W \in \mathbf{W}_{124}$ if and only if

$$
\sum_{i=1}^{j} \sum_{k=1}^{i} \Delta_{F k}^{(t, t+1)}(1) \geq \sum_{i=1}^{j} \sum_{k=1}^{i} \Delta_{G k}^{(t, t+1)}(1), \forall j=1, \ldots, n
$$

Eq. (9) is a third order direct dominance to be applied on distributions of mean income chang $€^{11}$ It is a weaker condition, allowing to order distributions when it is not possible to rank them according to proposition 6 and 7 .

\footnotetext{
${ }^{9}$ Note that the dominance condition stated in this proposition is equivalent to the last stage of the dominance condition state in proposition 4.

${ }^{10}$ Note that the dominance condition stated in this proposition is equivalent to the last stage of the dominance condition stated in proposition 5.

${ }^{11}$ The dominance conditions expressed in Proposition 6, 7 and 8 are equivalent respectively to "Type A", "Type B" and "Type C" dominance of absolute mobility profiles in Van Kerm $(2006 ; 2009)$.
} 
In summary, our dominance results are different from those derived by Bourguingon (2011). First, our conditions rest on the (sequential) comparison of quantile functions, whereas Bourguingon's (2011) rest on the (sequential) comparison of cdfs. Second, while Bourguingon's model builds on the assumption of same initial distributions of income, our model can be extended to evaluate growth processes taking place over different initial distributions of income. Still, our dominance results are related to Jenkins and Van Kerm's (2011) contribution. The essential difference is that we introduce a concern toward the horizontal inequality of the incidence of growth, which allows to obtain a set of new different dominance conditions. The introduction of horizontal inequality concerns is relevant not only because it allows to draw a more complete picture of the overall redistributive effect of growth, but also because it could help to explain possible divergences between anonymous and non-anonymous evaluations of growth.

\subsection{Welfare dominance and na-GIC}

In this section we study the relationship between our dominance conditions and the na-GIC (or Mobility Profile). The na-GIC, formally defined by: $\int_{0}^{1} \delta_{i}^{(t, t+1)}(p) d p / y_{t}\left(\frac{i}{n}\right)$, plots against each quantile of the initial distribution of income, the rate of income growth of that quantile, where the income refers to the same individuals in $t$ and $t+1$ (Grimm, 2007; Bourguignon, 2011; Jenkins and Van Kerm 2011).

Now, focussing on the family of $W \in \mathbf{W}$ and $W \in \mathbf{W}_{3}$, it is possible to note that if the dominance in eq. (2) or in eq. (5) holds for every $p$, than it must be the case that it holds if one integrates the quantile function of the income change from $p=0$ up to $p=1$, for all $p$ belonging to the same initial quantile. Hence, we have:

$$
W^{A} \geq W^{B} \Longrightarrow \int_{0}^{1} \delta_{A i}^{(t, t+1)}(p) d p \geq \int_{0}^{1} \delta_{B i}^{(t, t+1)}(p) d p \Longleftrightarrow \tilde{g}_{A i} \geq \tilde{g}_{B i}, \forall i=1, \ldots, n
$$

where $\tilde{g}_{A i}$ represents the absolute version of the na-GIC, which plots, against each quantile of the initial distribution, the level of income change of that quantile. We can summarize this discussion in the following remark.

Remark 1 a) For all $\Delta_{A}^{(t, t+1)}\left|y_{t}, \Delta_{B}^{(t, t+1)}\right| y_{t} \in D$, if $W_{A} \geq W_{B}, \forall W \in \mathbf{W}$ or $\forall W \in \mathbf{W}_{3}$ then $\tilde{g}_{A i} \geq \tilde{g}_{B i}, \forall i=1, \ldots, n$; b) For all $\Delta_{A}^{(t, t+1)}\left|y_{t}, \Delta_{B}^{(t, t+1)}\right| y_{t} \in D, W_{A} \geq W_{B}, \forall W \in \mathbf{W}_{4}$ if and only if $\tilde{g}_{A i} \geq \tilde{g}_{B i}, \forall i=1, \ldots, n$.

According to remark 1, first order dominance of na-GIC, weighted by the initial income of each quantile (or the absolute na-GIC), is a necessary condition, but not sufficient, for a normative dominance of two given growth processes, when the extent of growth or when both the extent of growth and its horizontal incidence matter. If we assume same initial distribution, this dominance reduces to first order na-GIC dominance. By contrast, when the dominance condition between two growth processes is based on social evaluation functions which are neutral to the horizontal incidence of growth, these dominance conditions arise to be equivalent to the absolute na-GIC dominance.

We now turn to consider the family of $W$ satisfying axioms 0,1 and $3^{*}$. Note that if the dominance in eq. (3) or in eq. (6) holds for every $p$, than it must be the case that it holds for 
$p=1$. Integrating sequentially the inverse cumulative distribution function up to $p=1$, the social evaluation dominance implies that:

$$
\sum_{i=1}^{k} \Delta_{A i}^{(t, t+1)}(1) \geq \sum_{i=1}^{k} \Delta_{B i}^{(t, t+1)}(1), \forall k=1, \ldots, n
$$

That is, at every stage we have to evaluate that the partial income growth mean is higher in one growth process than in the other. Thus

$$
W^{A} \geq W^{B} \Longrightarrow \sum_{i=1}^{k} \tilde{g}_{A i} \geq \sum_{i=1}^{k} \tilde{g}_{B i}, \forall k=1, \ldots, n
$$

We can summarize this discussion in the following remark.

Remark 2 a) For all $\Delta_{A}^{(t, t+1)}\left|y_{t}, \Delta_{B}^{(t, t+1)}\right| y_{t} \in D$, if $W_{A} \geq W_{B}, \forall W \in \mathbf{W}_{1}$ or $\forall W \in \mathbf{W}_{13^{*}}$ then $\left.\sum_{i=1}^{k} \tilde{g}_{A i} \geq \sum_{i=1}^{k} \tilde{g}_{B i}, \forall k=1, \ldots, n ; \mathbf{b}\right)$ For all $\Delta_{A}^{(t, t+1)}\left|y_{t}, \Delta_{B}^{(t, t+1)}\right| y_{t} \in D, W_{A} \geq W_{B}, \forall W \in$ $\mathbf{W}_{14}$ if and only if $\sum_{i=1}^{k} \tilde{g}_{A i} \geq \sum_{i=1}^{k} \tilde{g}_{B i}, \forall k=1, \ldots, n$.

According to remark 2, second order dominance of absolute na-GICs is a necessary, but not sufficient, condition for the dominance between growth processes, evaluated on the base of preferences expressing concern to the extent of growth and its vertical impact on the distribution of income or to the extent of growth and both its vertical and horizontal impact. If we assume same initial distributions, this dominance reduces to the second order na-GIC dominance. Further imposing axiom 4 , in addition to axiom 0 and 1 , we have an equivalence result.

\subsubsection{A measure of the progressivity of growth}

The dominance conditions established above provide robust but only partial rankings of growth processes. Complete rankings, instead, can be obtained adopting scalar measures and this is the aim of this section.

First, by considering the social evaluation function endorsing a social preference for the propoorness of growth and for horizontal inequality neutrality, hence satisfying axioms 0,1 and 4, an index of growth can be obtained by normalizing eq. (1) as follows:

$$
W^{*}=\frac{\sum_{i=1}^{n} q_{i} v_{i} \Delta_{i}^{(t, t+1)}(1)}{\sum_{i=1}^{n} q_{i} v_{i}}
$$

Eq. (12) evaluates growth accounting for both the size and the vertical redistribution impact of growth and can take both positive and negative values. Therefore, $W^{*}$ can be interpreted as a progressivity-sensitive measure of growth.

It is also possible to capture only the pure redistributional effect of an income transformation, in order to evaluate growth processes on the base of their ability to favor the growth of the initially 
poorest individuals as compared to the initially richest. This can be done using the following index:

$$
G=\frac{\sum_{i=1}^{n} q_{i} v_{i} \Delta^{(t, t+1)}(1)_{i}}{\sum_{i=1}^{n} q_{i} v_{i}}-\bar{W}^{*}
$$

Where $\bar{W}^{*}=\sum_{i=1}^{n} q_{i} \Delta_{i}^{(t, t+1)}(1)$ is is the overall mean income growth and can be interpreted as the growth every initial quantile group would experience in case of proportional growth. $G$ measures the relative distance between the effective process of growth and an hypothetical proportional proces: ${ }^{12}$. When $G>0$ we are in presence of a progressive growth process, that is growth is concentrated more among individuals ranked lower in the initial distribution of income; $G<0$ means that the growth process is regressive, that is income growth is concentrated more among the initially richer individuals; $G=0$ means that every individual experiences a proportional growth. Thus, this index can be interpreted as the gain (loss) in the social evaluation of growth due to its progressivity (regressivity)

\section{An empirical illustration}

\subsection{Data}

In this section we propose an empirical analysis of the distributional changes that took place in Italy during the last decade. In addition to show its empirical implementation, we use our framework to investigate the (very early) distributional impact of the economic crisis that took place in Italy in 2008 and is still going on.

We use the panel component of the "Survey on Household Income and Wealth" (SHIW). The SHIW is realized by the Bank of Italy every two years and is based on a representative sample of the Italian resident population. In particular, we consider eight years, from 2002 to 2010, and we divide the period in two time spells: 2002-2006 and 2006-2010. Hence, in order to grasp some information about the distributional impact of the crisis, we compare the growth process 2006-2010, encompassing the first phase of the economic crisis, to the immediately preceding 2002-2006 growth process. The unit of observation is the household, defined as all persons sharing the same dwelling. The variable of analysis is the household equivalent income ${ }^{14}$ expressed in terms of 2010 euro. Income includes all household earnings, transfers, pensions, and capital incomes, net of taxes and social security contributions. For each wave, we drop the richest and poorest $1 \%$ of the households in order to avoid outliers. We then obtain two samples of size: 2549 households for the period 2002/06 and 3384 for the period 2006/10.

We partition the initial distributions of income - 2002 for the first process and 2006 for the second - into 25 quantile groups, which we need in order to analyze the pro-poorness of growth. Each initial quantile group is then partitioned into quintiles of income change, which we need in order to analyze the horizontal incidence of growth 15 .

\footnotetext{
${ }^{12}$ A special case of this measure is provided by Jenkins and Van Kerm (2011; 2006) and Van Kerm (2006).

${ }^{13}$ See Demuynck and Van de gaer (2012) for alternative scalar measures to the evaluation of growth.

${ }^{14}$ We use the OECD equivalence scale given by the square root of the household size.

${ }^{15}$ See Peragine et al. (2011) for an analysis of growth in Italy using an equality of opportunity perspective.
} 
The yearly overall mean income growth rate computed for the panel component in the first period is $1,78 \%$; it is $0,034 \%$ in the second (see Table 1 ).

Table 1. Descriptive Statistics: Italy 2002/06 and 2006/10.

\begin{tabular}{lccc}
\hline Period & Sample size & Level income growth & $\begin{array}{c}\text { Proportionate income } \\
\text { growth }(\%)\end{array}$ \\
\hline $2002 / 2006$ & 2549 & 359.6225 & 1.78 \\
$2006 / 2010$ & 3384 & 6.98 & 0.034 \\
\hline
\end{tabular}

\subsection{Results}

We now turn to show the result of the tests presented in section 3 .

The dominance conditions from proposition 1 to proposition 5 are represented by sequential tests. Hence, given that we have divided the initial distribution of income into 25 quantile groups, we need to perform 25 tests for each of the propositions to prove the existence of a possible dominance between the growth processes compared. For the sake of brevity, the detailed results for every step are gathered in the appendix. Here, instead, we report a summary table (Table 2 panel (a)) indicating whether the dominance stated in each proposition holds and its sign.

We start from proposition 1, where only the size of growth and its direction (positive vs. negative growth) matter. As reported in Table 2 (a), although we compare a period of crisis to a period of growth, the two processes cannot be ordered. This happens because the condition imposed in this proposition is quite strong; it requires a first order dominance on the income change experienced by each individual within each initial quantile group. The impossibility of ordering the two processes derives from the fact that there exists first order dominance of the 2002/06 on the 2006/10 for many but not all the steps requires to validate the test (see Table 1A in Empirical Appendix).

Differently, when in this evaluation priority is given to the growth experienced by those individuals initially ranked lowest, as in proposition 2 and 3, the first process dominates the second. In fact, the existence of first order dominance of the 2002/06 over the 2006/10 growth process can be proved at every step of the sequential aggregation (see Tables 2A and 3A in Appendix). Hence, the 2002/06 episode can be considered as more socially desirable than the 2006/10 when, in addition to the extent of growth, its "non-anonymous" pro-poorness plays a role in the evaluation procedure.

We then move to compare the two processes on the base of a social preference encompassing only a concern toward the direction and the horizontal inequality of growth. This is the case of the test presented in proposition 4 . This test deals with the comparison of cumulated distributions of income change between the 2002/06 and 2006/10, for each of the 25 initial quantile groups. However, these cumulated distributions overlap in some of the steps of the proposition, making impossible to establish a clear rank (see Table 4A in Appendix).

By contrast, a clear cut picture can be obtained looking at the results of proposition 5 . In fact, the first growth process arises to be more socially preferable than the second, implying that the non-anonymous income transformation process is more desirable for the first four-year period, when concerns for the extent of growth and for both vertical and horizontal incidence are introduced.

The conditions applied till now are aimed at capturing and explaining both aspects of the redistributive effect of growth, that is its vertical and horizontal inequality. Although quite restrictive, the 2002/06 growth process arises to dominate the 2006/10 for most of these conditions. It is possible to obtain further support to this result by applying the less restrictive dominance presented in proposition 6, 7 and 8. Less restrictive conditions derive when we impose horizontal inequality 
neutrality and we only care about the impact on the vertical redistribution of growth according to the non-anonymous approach. The results are reported in Table 2 (b) and in Figure 1 (a) and Figure 2 (a) (see also Table 6A, 7A, 8A in the Empirical Appendix)

Table 2. (a) Results of the tests presented in Proposition 1, 2, 3, 4 and 5: 2002/06 vs. 2006/10. (b) Results of the tests presented in Proposition 6, 7 and 8: 2002/06 vs. 2006/10;

(a)

\begin{tabular}{lc}
\hline Proposition & $2002 / 06$ vs. 2006/10 \\
\hline 1 & No \\
2 & $>$ \\
3 & $>$ \\
4 & No \\
5 & $>$ \\
\hline
\end{tabular}

(b)

\begin{tabular}{lc}
\hline Proposition & $2002 / 06$ vs. 2006/10 \\
\hline 6 & No \\
7 & $>$ \\
8 & $>$ \\
\hline
\end{tabular}

Notes: "NO" the two processes cannot be ordered; ">" the 2002/06 process dominates the 2006/10.

Figure 1 (a) reports the curves obtained applying proposition 6. They are constructed by plotting the level of income change experienced by the individuals belonging to each of the 25 initial quantile groups, therefore they can be considered as the absolute version of the non-anonymous GIC (or absolute "mobility profiles" according to Van Kerm, 2009 and Jenkins and Van Kerm, 2011). Although we cannot state that a clear dominance exists between the two processes since they overlap in some points of the distribution, some interesting features arise. First, looking at the overall process, it is straightforward to notice that the non-anonymous growth dynamics under scrutiny are pro-poor; in fact, the two absolute naGICs are characterized by the same decreasing pattern implying that growth favors more the initially poorest individuals in both period considered. Second, the curve representing the 2002/06 lies almost always above the curve representing the $2006 / 10$, however there are three initial quantile groups in the lowest half of the distribution, namely 4, 6 and 10, and one initial quantile group in the upper half of the distribution, namely the 24 , where the $2006 / 10$ slightly dominates the $2002 / 06$. Hence, from a comparative point of view it is not clear what part of the distribution has been affected more by the crisis. Although it could be argued that, among them, it is the richest quantile group (group 24) to lose less under the 2006/10 than the 2002/06, since as it is possible to see from Figure 1 (a) the difference between the two curves for this part of the distribution is higher than the difference between the two curves for the initial quantile group 4, 6 and 10 together.

Figure 1. (a) Proposition 6: Italy, 2002/06 vs. 2006/10; (b) Absolute GIC: Italy, 2002/06 vs. 2006/10.

(a)

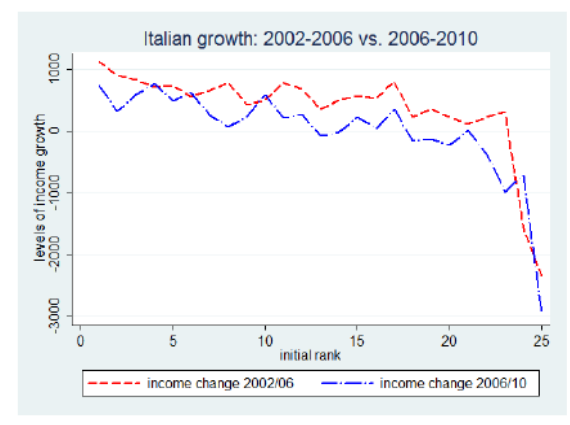

(b)

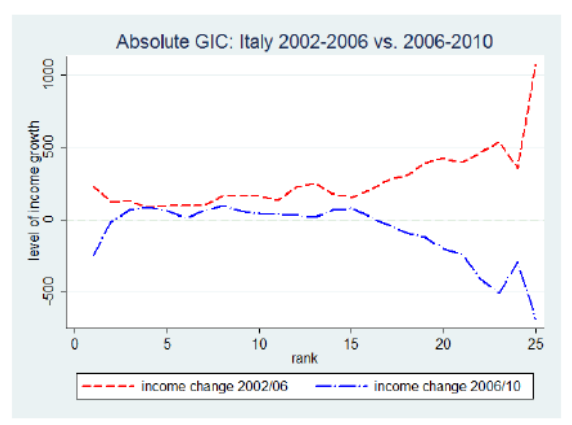


Figure 2. (a) Proposition 7: Italy, 2002/06 vs. 2006/10; (b) Cumulated Absolute GIC: Italy, $2002 / 06$ vs. $2006 / 10$.

(a)

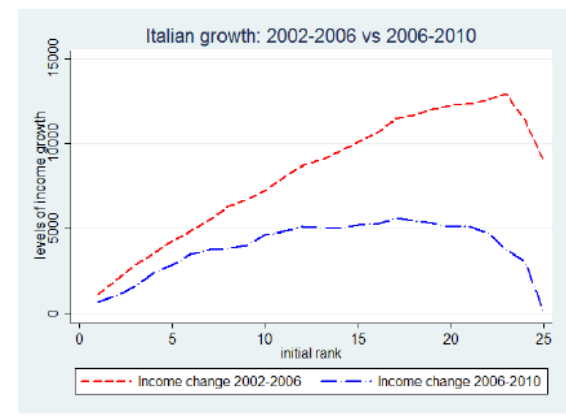

(b)

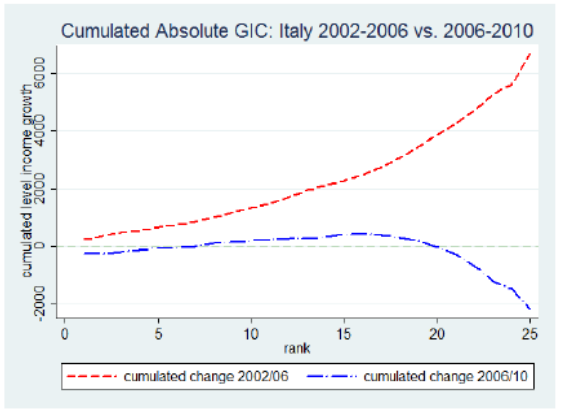

In order to grasp the relevance of adopting our framework as a complementary tool to the standard analysis of growth, it is interesting to compare the curves in Proposition 6 which are absolute version of the non-anonymous growth incidence curve, with the absolute version of the GIC reported in Figure 1(b). The divergence between our approach and the standard approach is striking. First the absolute GIC for the first process (Figure 1 (b)) lies always above the second process implying that there is a clear dominance of the 2002/06 over the 2006/10 growth process. Second, the trend shown by each single curve is very different form that arising in Figure 1 (a), in particular for the first process. Notice, in fact, that when we adopt an anonymous approach, every percentile of the initial distribution experiences a positive change in the level of income, but this change appears strongly regressive. Whereas, under the second process, the trend is always progressive, but now the most part of the distribution, in particular the richest quantiles experience negative growth. The implications in terms of effect of the crisis seems also to be reverted by this graph. In fact the divergence between the two processes is impressive for the richest individuals. Hence, the employment of the non-anonymous approach to evaluate growth may be a useful complement to the standard approach. As shown clearly for the first process, an absolute GIC with a regressive pattern may be associated to an absolute naGIC with a progressive one, which is realistic and witnesses how the focus of the two approaches differs: the GIC is concerned with the income growth of each part of the distribution, independently of the individuals sitting in that part, whereas the naGIC is concerned with the income dynamic of each individual in a distribution (or income mobility).

We now turn to apply the dominance condition of proposition 7 , which is based on the size and on the pro-poorness features of growth. Figure 2 (a) reports the curves derived from proposition 7 , they plot the cumulated sum of the income change experienced by each initial quantile (see also Table 7A in the appendix). The comparison between the two dynamics is now very clear. Both curves increase steeply up to the 20th quantile, becoming decreasing for the richest quantiles. However, the curve relative to the first process is always higher than the second implying that the $2002 / 06$ dominates the 2006/10 when the social evaluation of growth is based on efficiency and propoorness features of growth. They also show a concave shape which appears to be more pronounced in the second process, implying that this process might have been more effective in terms of vertical impact. Thus, it could be inferred that the dominance of the 2002/06 growth over the 2006/10 is mostly due to the higher absolute growth, which appears to compensate for the lower degree of concavity. 
Again the difference between our approach and the standard non-anonymous one appears from the comparison of the curves reported in Figure 2 (a) with the curves reported in Figure 2 (b). The sign of the dominance is the same (2002/06 dominates the 2006/10) however, the specific shape of each curve is quite different, in particular for the first growth episode ${ }^{16}$

The results obtained implementing proposition 6 and 7 are confirmed by the employment of the dominance condition in proposition 8 (see table $8 \mathrm{~A}$ in the appendix) and by the adoption of the scalar measures defined in eq. (12) and (13). The two indices ${ }^{17}$ give us a clear information about the complete ranking and the features of each income dynamic. In particular, while the index in eq. (12), the progressivity-sensitive measure of growth, reflects both size and vertical redistributive effect of growth, the index in eq. (13) is only concerned with the latter component, hence they may generate opposing ranking. Their results are reported in Table 2. As concerned the progressivitysensitive index of growth, it arises to be higher for the first growth process than for the second. The complete ranking provided clearly indicates that the 2002/06 growth process dominates the 2006/10, when considering both the extent of growth and its vertical impact. By contrast, the complete ranking provided by eq. (13) indicates that the second process dominates the first one if only the vertical redistributive effect is taken into consideration. In summary, the larger value of $W^{*}$ for the first period reflects the grater average growth in this period which compensate for the lower return to progressivity. Whereas, the higher value of $G$ for the second period states that although a lower growth in level, the second process shows higher degree of return to progressivity (in absolute terms). However, note that the order of dominance between the two processes, provided by $G$ is reverted when relative changes, instead of absolute changes, are considered (Table 3).

Table 3. Indices of growth: Italy 2002/06 and 2006/10.

\begin{tabular}{|c|c|c|c|}
\hline & \multicolumn{3}{|c|}{ Level income growth } \\
\hline Period & $\begin{array}{l}\text { Progressivity sensitive } \\
\text { growth }\left(W^{*}\right)\end{array}$ & $\begin{array}{c}\text { Return to } \\
\text { progressivity }(G)\end{array}$ & $\begin{array}{l}\text { Mean income } \\
\text { growth }\left(\gamma_{a}\right)\end{array}$ \\
\hline $2002 / 06$ & 657.0134 & 298.2144 & 359.873 \\
\hline $2006 / 10$ & 324.6083 & 320.5969 & 6.98 \\
\hline & \multicolumn{3}{|c|}{ Proportionate income growth (\%) } \\
\hline Period & $\begin{array}{l}\text { Progressivity sensitive } \\
\text { growth }\left(W^{*}\right)\end{array}$ & $\begin{array}{c}\text { Return to } \\
\text { progressivity }(G)\end{array}$ & $\begin{array}{l}\text { Mean income } \\
\text { growth }\left(\gamma_{r}\right)\end{array}$ \\
\hline $2002 / 06$ & 7.001 & 2.828 & 1.78 \\
\hline $2006 / 10$ & 3.722 & 1.984 & 0.0034 \\
\hline
\end{tabular}

$$
\text { Notes: } \gamma_{r}=\frac{\mu\left(y_{t+1}\right)-\mu\left(y_{t}\right)}{\mu\left(y_{t}\right)} ; \gamma_{a}=\mu\left(y_{t+1}\right)-\mu\left(y_{t}\right)
$$

In sum, the results of the implementation of the partial and complete dominance conditions introduced in section 3 - with the only exception of the index of progressivity - witness the severe impact of the crisis according to different features of growth. It can be argued that the increase in unemployment caused by the crisis is among the main determinants of the negative performance of the growth process in 2006/10 with respect to the growth process in 2002/06. In particular, the flexibility introduced in the Italian labor market at the end of the 90s could have played a relevant role. In fact, job flexibility generates more opportunities during periods of positive growth, as also

\footnotetext{
${ }^{16}$ For an additional comparison see Figure $1 \mathrm{~A}$ and $3 \mathrm{~A}$ reporting, the relative version of the curve in proposition 6 and 7 , and Figure $2 \mathrm{~A}$ and $4 \mathrm{~A}$ reporting the relative version of the GIC and cumulative GIC.

${ }^{17}$ We adopt the following parametrization: $v_{i}=2\left(1-\frac{i}{n}\right)$.
} 
shown in our analysis for the growth episode 2002/06, increasing the real income of most individuals in the population. This trend is then reverted with the advent of the crisis. In fact, the workers who benefit from flexibility, at the beginning of the decade, are the same to be exposed to the risk of being fired and were effectively fired because of the crisis, with the consequent huge fall in their disposable income ${ }^{18}$

\section{Conclusions}

Recent contributions show the need to modify standard frameworks for measuring the distributional effect of growth, in order to take into account the possibility of the identity of individuals, hence their reshuffling among income classes. In this work we have provided a normative approach to rank growth processes when these further aspects are a matter of concern. We have adopted a bi-dimensional framework, where the two dimensions are respectively the initial rank of individuals and their income transformation. We have proposed to aggregate these information according to a rank dependent approach, which makes it possible to account for the size of growth and its vertical and horizontal impact. We have provided partial dominance conditions for ordering growth processes and we have shown how these conditions relate to na-GIC. Moreover, we have proposed two classes of indices: one aimed at capturing at the same time the extent of growth and its vertical redistributive effect; the other aimed at isolating the latter component. We have then applied our framework for the assessment of the distributional impact of growth in Italy in the last decade, with a particular focus on the effect of the recent economic crisis. Specifically, we have considered four of the most recent available waves to compare the 2002-2006 growth episode against the 2006-2010. The results obtained in our analysis allow us to argue that the economic crisis has been a negative event not only from a macro-perspective, but it also lead to a worse performance of the Italian income dynamic from a micro-perspective.

Our empirical illustration witnesses that the measurement framework we have introduced can usefully complement existing tools for the evaluation of growth. In particular, it stems out that further research for the understanding of the underlying relationship between anonymous and nonanonymous growth (or income mobility) seems to be a promising path. Another field of application of our framework is the analysis of tax-benefit reforms. Their distributional aspects are, in general, evaluated in terms of income inequality reduction. At the same time, we argue that the evaluation and comparison of these reforms in terms of their impact on the individual income trajectories, with the help of the tools developed in this paper, might be potentially relevant.

\section{References}

[1] Aaberge, R. (2001). Axiomatic Characterization of the Gini Coefficient and Lorenz Curve Orderings, Journal of Economic Theory, 101, 115-132.

[2] Banca d'Italia (2008) Supplemento al Bollettino Statistico Note Metodologiche e informazioni Statistiche, I Bilanci delle Famiglie Italiane nell'Anno 2006, Anno XVIII Numero 7 - 28 Gennaio 2008.

[3] Banca d'Italia (2012) Supplemento al Bollettino Statistico Note Metodologiche e informazioni Statistiche, I Bilanci delle Famiglie Italiane nell'Anno 2010, Anno XXII - 25 Gennaio 2012.

\footnotetext{
${ }^{18}$ See on this Boeri and Garibaldi (2007).
} 
[4] Bénabou, R., Ok, E. (2001). Mobility as progressivity: ranking income processes according to equality of opportunity, NBER Working Paper No. 8431

[5] Boeri, T., Garibaldi, P. (2007). Two Tier Reforms of Employment Protection: a Honeymoon Effect?, Economic Journal, Royal Economic Society, vol. 117(521), 357-385.

[6] Bourguignon, F. (2003). The Growth Elasticity of Poverty Reduction: Explaining Heterogeneity across Countries and Time Periods. In: Eicher, T., Turnovsky, S. Inequality and growth: theory and policy implications. MIT Press, Cambridge.

[7] Bourguignon, F. (2004). The Poverty-Growth-Inequality Triangle. Indian Council for Research on International Economic Relations, Working Paper N.125.

[8] Bourguignon, F. (2011). Non-anonymous Growth Incidence Curves, Income Mobility and Social Welfare Dominance, Journal of Economic Inequality, 9(4), 9(4), 605-627.

[9] Chambaz, C., Maurin, E. (1998). Atkinson and Bourguignon's Dominance Criteria: Extended and Applied to the Measurement of Poverty in France, Review of Income and Wealth, 44, 4.

[10] Datt, G., Ravallion, M. (1992). Growth and redistribution components of changes in poverty measures: a decomposition with applications to Brazil and India in the 1980s, Journal of Development Economics, 38, 275-95.

[11] Demuynck, T., Van de gaer, D. (2012). Inequality Adjusted Income Growth, Economica, 79, 747-765.

[12] Donaldson, D., Weymark, J. (1980). A Single-Parameter Generalization of the Gini Indices of Inequality, Journal of Economic Theory, 22, 67-86

[13] Duclos, J. (2009). What is pro-poor?, Social Choice and Welfare, 32, 37-58.

[14] Essama-Nssah, B. (2005). A Unified Framework for Pro-Poor Growth Analysis, Economics Letters, 89, 216-221.

[15] Essama-Nssah, B., Lambert, P. (2009). Measuring Pro-poorness: a Unifying Approach with New Results, Review of Income and Wealth, 55, 3, 752-778.

[16] Ferreira, F.H.G. (2010), Distributions in motion. Economic growth, inequality, and poverty dynamics, World Bank, Policy research working paper working paper N. 5424.

[17] Grimm, M. (2007). Removing the anonymity axiom in assessing pro-poor growth, Journal of Economic Inequality, 5(2), 179-197.

[18] Jenkins, S., Van Kern, P. (2006). Trends in income inequality, pro-poor income growth and income mobility, Oxford Economic Papers, 58, 3, 531-48.

[19] Jenkins, S., Van Kerm, P. (2011). Trends in individual income growth: measurement methods and British evidence, IZA DP. No. 5510.

[20] Kakwani, N., Pernia, E. (2000). What is Pro-poor Growth?, Asian Development Review, 18, $1-16$. 
[21] Kakwani, N., Son, H. (2008). Poverty Equivalent Growth Rate, Review of Income and Wealth, $54,4,643-655$.

[22] Kraay, A. (2006). When is Growth Pro-poor? Evidence From a Panel of Countries, Journal of Development Economics, 80, 1, 198-227.

[23] Peragine, V. (2002). Opportunity Egalitarianism and Income Inequality: the rank dependent approach, Mathematical Social Sciences, 44, 45-64.

[24] Peragine, V., Brunori, P., Palmisano, F. (2011). Economic growth and equality of opportunity, ECINEQ WP n. 232.

[25] Ravallion, M., Chen, S. (2003). Measuring pro-poor growth, Economics Letters, 78, 1, 93-9.

[26] Son, H.H. (2004). A note on pro-poor growth, Economics Letters, 82, 3, 307-314.

[27] Van Kerm, P. (2006). Comparisons of income mobility profiles, IRISS Working Paper 2006/03, CEPS/INSTEAD, Differdange, Luxembourg.

[28] Van Kerm, P. (2009). Income mobility profiles, Economic Letters, 102, 2, 93-95.

[29] Yaari, M. (1988). A Controversial Proposal Concerning Inequality Measurement, Journal of Economic Theory, 44, 381-397.

[30] Zheng, B. (2010). Consistent Comparison of Pro-poor Growth, Social Choice and Welfare, 37, 61-79.

[31] Zoli, C. (2000). Inverse Sequential Stochastic Dominance: Rank-Dependent Welfare, Deprivation and Poverty Measurement, Discussion Paper in Economics, No. 00/11, University of Nottingham.

\section{Theoretical Appendix}

\section{Proof of Proposition 1}

We want to find a necessary and sufficient condition for

$$
\Delta W=\sum_{i=1}^{n} \int_{0}^{1} v_{i}(p)\left[\delta_{A i}^{(t, t+1)}(p)-\delta_{B i}^{(t, t+1)}(p)\right] d p \geq 0, \forall W \in \mathbf{W}
$$

Sufficiency clearly derives from the fact that since $v_{i}(p) \geq 0, \forall p \in[0,1]$ and $\forall i=1,2, \ldots, n$, $\delta_{A i}^{(t, t+1)}(p)-\delta_{A i}^{(t, t+1)}(p) \geq 0, \forall p \in[0,1]$ and $\forall i=1,2, \ldots, n$, implies $\Delta W \geq 0$.

For the necessity, suppose for a contradiction that $\Delta W \geq 0, \forall W \in \mathbf{W}_{1}$, but there is a quantile group $h \in\{1, \ldots, n\}$ and an interval $I \equiv[a, b] \subseteq[0,1]$ such that $\delta_{A h}^{(t, t+1)}(p)-\delta_{B h}^{(t, t+1)}(p)<0, \forall p \in I$. Now select a set of functions $\left\{v_{i}(p)\right\}_{i \in\{1, \ldots, m\}}$ such that $v_{i}(p) \searrow 0, \forall i \neq h$ and $v_{h}(p) \searrow 0$, $\forall p \in[0,1] \backslash I$. in this case $\Delta W$ would reduce to $\int_{a}^{b} v_{h}(p)\left[\delta_{A h}^{(t, t+1)}(p)-\delta_{B h}^{(t, t+1)}(p)\right] d p<0$, a contradiction. QED 


\section{Proof of Proposition 2}

Before proving this proposition we need to prove the following lemma.

Lemma $1 \sum_{k=1}^{n} v_{k} w_{k} \geq 0$ for all sets of numbers $\left\{v_{k}\right\}$ such that $v_{k} \geq v_{k+1} \geq 0 \forall k \in\{1, \ldots, n\}$ if and only if $\sum_{i=1}^{k} w_{i} \geq 0, \forall k \in\{1, \ldots, n\}$.

Proof. Applying Abel's decomposition: $\sum_{k=1}^{n} v_{k} w_{k}=\sum_{k=1}^{n}\left(v_{k}-v_{k+1}\right) \sum_{i=1}^{k} w_{i}$. If $\sum_{i=1}^{k} w_{i} \geq 0$, $\forall k \in\{1, \ldots, n\}$, then $\sum_{k=1}^{n} v_{k} w_{k} \geq 0 . \quad$ As for the necessity part, suppose that $\sum_{k=1}^{n} v_{k} w_{k} \geq 0$ for all sets of numbers $\left\{v_{k}\right\}$ such that $v_{k} \geq v_{k+1} \geq 0$, but $\exists j \in\{1, \ldots, n\}: \sum_{i=1}^{j} w_{i}<0$. Consider what happens when $\left(v_{k}-v_{k+1}\right) \searrow 0, \forall k \neq j$. We obtain that $\sum_{k=1}^{n} v_{k} w_{k} \longrightarrow\left(v_{j}-v_{j+1}\right) \sum_{i=1}^{j} w_{i}<0$, a contradiction.

We now want to find a necessary and sufficient condition for

$$
\Delta W=\sum_{i=1}^{n} \int_{0}^{1} v_{i}(p)\left[\delta_{A i}^{(t, t+1)}(p)-\delta_{B i}^{(t, t+1)}(p)\right] d p \geq 0, \forall W \in \mathbf{W}_{1}
$$

Sufficiency can be shown as follows. First, reverse the order of integration and summation, such that

$$
\Delta W=\int_{0}^{1} \sum_{i=1}^{n} v_{i}(p)\left[\delta_{A i}^{(t, t+1)}(p)-\delta_{B i}^{(t, t+1)}(p)\right] d p \geq 0
$$

Letting $S_{i}(p)=\delta_{A i}^{(t, t+1)}(p)-\delta_{B i}^{(t, t+1)}(p)$ and rewriting (16):

$$
\Delta W=\int_{0}^{1} \sum_{i=1}^{n} v_{i}(p) S_{i}(p) d p \geq 0
$$

Since $v_{i}(p) \geq v_{i+1}(p) \geq 0, \forall i=1, \ldots, n-1$ and $\forall p \in[0,1]$, we can apply Lemma 1 and obtain that $\sum_{i=1}^{n} v_{i}(p) S_{i}(p) \geq 0$ if and only if $\sum_{i=1}^{k} S_{i}(p) \geq 0, \forall k=1, \ldots, n$ and $\forall p \in[0,1]$. It follows that $\sum_{i=1}^{n} v_{i}(p) S_{i}(p) \geq 0, \forall p \in[0,1]$, implies that, integrating with respect to $p, \int_{0}^{1} \sum_{i=1}^{n} v_{i}(p) S_{i}(p) d p \geq 0$.

For the necessity, suppose for a contradiction that $\Delta W \geq 0, \forall W \in \mathbf{W}_{12}$, but there is an initial quantile $h \in\{1, \ldots, n\}$ and an interval $I \equiv[a, b] \subseteq[0,1]$ such that $\sum_{i=1}^{h} S_{i}(p)<0, \forall p \in I$. Now, 
applying Lemma 1 , there exists a set of functions $\left\{v_{i}(p) \geq 0\right\}:[0,1] \longrightarrow \Re_{+}, i=1, \ldots, n$, such that $\sum_{i=1}^{n} v_{i}(p) S_{i}(p)<0, \forall p \in I$. Writing $\sum_{i=1}^{n} v_{i}(p) S_{i}(p)=T(p), \Delta W\left(\Delta^{(t, t+1)} \mid y_{t}\right)$ reduces to $\int_{0}^{1} T(p) d p$, where $T(p)<0, \forall p \in I$. Selecting a set of function $T(p)$, such that $T(p) \longrightarrow 0$, $\forall p \in[0,1] \backslash I, \Delta W$ would reduce to $\int_{a}^{b} T(p) d p<0$, a contradiction. QED

\section{Proof of Proposition 3}

We want to find a necessary and sufficient condition for

$$
\Delta W=\sum_{i=1}^{n} \int_{0}^{1} v_{i}(p)\left[\delta_{A i}^{(t, t+1)}(p)-\delta_{B i}^{(t, t+1)}(p)\right] d p \geq 0, \forall W \in \mathbf{W}_{12}
$$

For the sufficiency, note that if $v_{i}(p)$ satisfies axiom 0,1 and 2 , we can revert the order of integration and summation and apply Abel's decomposition to obtain: $\Delta W=\int_{0}^{1}\left[\sum_{i=1}^{n}\left(v_{i}(p)-v_{i+1}(p)\right) \sum_{k=1}^{i} S_{k}(p)\right] d p$, where $S_{k}(p)=\delta_{A k}^{(t, t+1)}(p)-\delta_{B k}^{(t, t+1)}(p)$. Let $v_{i}(p)-v_{i+1}(p)=\omega_{i}(p)$ and $\sum_{k=1}^{i} S_{k}(p)=\kappa_{i}(p)$, by axiom $3 \omega_{i}(p)>\omega_{i+1}(p), \forall i=1, \ldots, n-1, \forall p \in[0,1]$. We can apply Lemma 1 to get $\sum_{i=1}^{n} \omega_{i}(p) \kappa_{i}(p) \geq 0$ if and only if $\sum_{i=1}^{j} \kappa_{i}(p) \geq 0, \forall j=1, \ldots, n, \forall p \in[0,1]$. If $\sum_{i=1}^{n} \omega_{i}(p) \kappa_{i}(p) \geq 0$, $\forall p \in[0,1]$, implies that $\Delta W=\int_{0}^{1} \sum_{i=1}^{n} \omega_{i}(p) \kappa_{i}(p) d p \geq 0$. Thus $\sum_{i=1}^{j} \sum_{k=1}^{i} \delta_{A i}^{(t, t+1)}(p)-\delta_{B i}^{(t, t+1)}(p)$, $\forall j=1, \ldots, n, \forall p \in[0,1]$ is sufficient for $\Delta W \geq 0$.

For the necessity, let $T(p) \equiv \sum_{i=1}^{n} \omega_{i}(p) \kappa_{i}(p)$, we can write the following $\Delta W=\int_{0}^{1} T(p) d p$. Suppose that $\Delta W \geq 0, \forall W \in \mathbf{W}_{123}$, but $\exists h=1, \ldots, n$ and $\exists I \equiv[a, b] \subseteq[0,1]$ such that $\sum_{i=1}^{h} \kappa_{i}(p)<$ $0, \forall p \in I$. Then by Lemma $1 \exists$ a set of functions $\omega_{i}(p):[0,1] \longrightarrow \Re_{+}, i=1, \ldots, n$, such that $T(p)<0, \forall p \in I$. We can chose a function $T(p)$ such that $T(p) \longrightarrow 0, \forall p \in[0,1] \backslash I$, thus $\Delta W$ would reduce to $\int_{a}^{b} T(p) d p<0$, a contradiction. QED

\section{Proof of Proposition 4}

We want to find a necessary and sufficient condition for

$$
\Delta W=\sum_{i=1}^{n} \int_{0}^{1} v_{i}(p)\left[\delta_{A i}^{(t, t+1)}(p)-\delta_{B i}^{(t, t+1)}(p)\right] d p \geq 0, \forall W \in \mathbf{W}_{3}
$$


For the sufficiency, let $S_{i}(p)=\delta_{A i}^{(t, t+1)}(p)-\delta_{B i}^{(t, t+1)}(p)$, we can integrate eq. (19) by parts to get:

$$
\Delta W=\sum_{i=1}^{n}\left[v_{i}(1) \int_{0}^{1} S_{i}(p) d p\right]-\sum_{i=1}^{n} \int_{0}^{1} v_{i}^{\prime}(p) \int_{0}^{p} S_{i}(q) d q d p
$$

It follows that

$$
\int_{0}^{p} \delta_{A i}^{(t, t+1)}(q)-\delta_{B i}^{(t, t+1)}(q) d q \geq 0, \forall p \in[0,1], \forall i=1, \ldots, n
$$

is sufficient for welfare dominance, since by axiom $0 v_{i}(p) \geq 0$ eq. (21) implies the positivity of $v_{i}(1) \int_{0}^{1} S_{i}(p) d p$ and by axiom $3 v_{i}^{\prime}(p) \leq 0$ it implies the negativity of the second term of eq. (20). It follows that $\Delta W \geq 0$.

For the necessity, suppose that $\Delta W \geq 0$, but $\exists h=1, \ldots, n$ and $\exists I \equiv[a, b] \subseteq[0,1]$ such that $\int_{0}^{p} \delta_{A h}^{(t, t+1)}(q)-\delta_{B h}^{(t, t+1)}(q) d q<0, \forall p \in I$. Let $T_{i}(p)=v_{i}^{\prime}(p) \int_{0}^{p} \delta_{A i}^{(t, t+1)}(q)-\delta_{B i}^{(t, t+1)}(q) d q$, $\forall i=1, \ldots, n, \forall p \in[0,1]$. We can chose a set of functions $T_{i}(p)$ such that $v_{i}^{\prime}(p) \longrightarrow 0, \forall i \neq h$ and $T(p) \longrightarrow 0, \forall p \in[0,1] \backslash I$ and we can chose a combination of $v_{i}(1)$ and $S_{i}(p)$ such that $\sum_{i=1}^{n} v_{i}(1) \int_{0}^{1} S_{i}(p) d p=0$. Then, $\Delta W$ would reduce to $-\int_{a}^{b} T_{h}(p) d p \leq 0$, a contradiction. QED

\section{Proof of Proposition 5}

We want to find a necessary and sufficient condition for

$$
\Delta W=\sum_{i=1}^{n} \int_{0}^{1} v_{i}(p)\left[\delta_{A i}^{(t, t+1)}(p)-\delta_{B i}^{(t, t+1)}(p)\right] d p \geq 0, \forall W \in \mathbf{W}_{13^{*}}
$$

Sufficiency can be shown as follows. First, reverse the order of integration and summation, such that

$$
\Delta W=\int_{0}^{1} \sum_{i=1}^{n} v_{i}(p)\left[\delta_{A i}^{(t, t+1)}(p)-\delta_{B i}^{(t, t+1)}(p)\right] d p \geq 0
$$

Letting $S_{i}(p)=\delta_{A i}^{(t, t+1)}(p)-\delta_{B i}^{(t, t+1)}(p)$ and $\varepsilon_{i}(p)=v_{i}(p)-v_{i+1}(p) \geq 0, \forall i=1, \ldots, n-1$ and $\forall p \in[0,1]$, by axiom 0 and application of Abel's decomposition we can rewrite eq. (23):

$$
\Delta W=\int_{0}^{1} \sum_{i=1}^{n}\left(\varepsilon_{i}(p) \sum_{j=1}^{i} S_{j}(p)\right) d p \geq 0
$$

Integrating by parts

$$
\Delta W=\sum_{i=1}^{n}\left(\varepsilon_{i}(1) \sum_{j=1}^{i} \int_{0}^{1} S_{j}(p) d p\right)-
$$




$$
\int_{0}^{1} \sum_{i=1}^{n}\left(\varepsilon_{i}^{\prime}(p) \sum_{j=1}^{i} \int_{0}^{p} S_{j}(q) d q\right) d p \geq 0
$$

By axiom $1 \varepsilon_{i}(1) \geq 0$, by axiom $3^{*} \varepsilon_{i}^{\prime}(p) \leq 0, \forall i=1, \ldots, n$ and $\forall p \in[0,1]$, it follows that $\sum_{j=1}^{i} \int_{0}^{p} S_{j}(q) d q \geq 0, \forall i=1, \ldots, n$ and $\forall p \in[0,1]$ implies $\Delta W \geq 0$.

For the necessity, write eq. (25) as follows:

$$
\begin{gathered}
\Delta W=\sum_{i=1}^{n}\left(\varepsilon_{i}(1) \sum_{j=1}^{i} \int_{0}^{1} S_{j}(p) d p\right)+ \\
\int_{0}^{1} \sum_{i=1}^{n}\left(\left(\omega_{i}(p)\right) \sum_{j=1}^{i} \int_{0}^{p} S_{j}(q) d q\right) d p \geq 0
\end{gathered}
$$

with $\omega_{i}(p)=-\varepsilon_{i}^{\prime}(p)$. Now, suppose for a contradiction that $\Delta W \geq 0$, but $\exists h \in\{1, \ldots, n\}$ and an interval $I \equiv[a, b] \subseteq[0,1]$ such that $\sum_{j=1}^{h} \int_{0}^{p} S_{j}(q) d q<0, \forall p \in I$. Now, given that $\left\{\omega_{i}(p) \geq 0\right\}_{i \in\{1, \ldots, n\}}$, applying Lemma 1 in Chambaz and Maurin (1998) we will have $\sum_{i=1}^{n} \omega_{i}(p)\left(\sum_{j=1}^{i} \int_{0}^{p} S_{j}(q) d q\right)<0$ $\forall p \in I$. Now let $R(p)=\sum_{i=1}^{n} \omega_{i}(p)\left(\sum_{i=1}^{i} \int_{0}^{p} S_{i}(q) d q\right)$, then $R(p)<0, \forall p \in I$. Then, $\Delta W=$ $\sum_{i=1}^{n}\left(\varepsilon_{i}(1) \sum_{j=1}^{i} \int_{0}^{1} S_{j}(p) d p\right)+\int_{0}^{1} R(p) d p$

Now choosing $R(p)$ such that $R(p) \longrightarrow 0$ for some $p \in[0,1] \backslash I$,

$$
\Delta W=\sum_{i=1}^{n}\left(\varepsilon_{i}(1) \sum_{j=1}^{i} \int_{0}^{1} S_{j}(p) d p\right)-\int_{a}^{b} R(p) d p
$$

We can choose a combination of $\varepsilon_{i}(1)$ and $\sum_{j=1}^{i} \int_{0}^{1} S_{j}(p) d p$, such that $\sum_{i=1}^{n}\left(\varepsilon_{i}(1) \sum_{j=1}^{i} \int_{0}^{1} S_{j}(p) d p\right)=$ 0, then $\Delta W=\int_{a}^{b} R(p) d p \leq 0$, a desired contradiction. QED.

\section{Proof of Proposition 6}

We want to find a necessary and sufficient condition for

$$
\Delta W=\sum_{i=1}^{n} \int_{0}^{1} v_{i}(p)\left[\delta_{A i}^{(t, t+1)}(p)-\delta_{B i}^{(t, t+1)}(p)\right] d p \geq 0, \forall W \in \mathbf{W}_{4}
$$


For the sufficiency, by axiom $4 v_{i}(p)=\beta_{i}, \forall p \in[0,1]$ and $\forall i=1,2, \ldots, n$, therefore we can write eq. (27) as follows:

$$
\begin{aligned}
\Delta W & =\sum_{i=1}^{n} \beta_{i} \int_{0}^{1}\left[\delta_{A i}^{(t, t+1)}(p)-\delta_{B i}^{(t, t+1)}(p)\right] d p= \\
& =\sum_{i=1}^{n} \beta_{i}\left[\Delta_{A i}^{(t, t+1)}(1)-\Delta_{B i}^{(t, t+1)}(1)\right] \geq 0
\end{aligned}
$$

by axiom $0 v_{i}(p)=\beta_{i} \geq 0, \forall p \in[0,1]$ and $\forall i=1,2, \ldots, n, \Delta W \geq 0$ if $\Delta_{A i}^{(t, t+1)}(1)-\Delta_{B i}^{(t, t+1)}(1) \geq 0$, $\forall i=1, \ldots, n$.

For the necessity, suppose that

$$
\Delta W=\sum_{i=1}^{n} \beta_{i}\left[\Delta_{A i}^{(t, t+1)}(1)-\Delta_{B i}^{(t, t+1)}(1)\right] \geq 0
$$

but $\exists k=1, \ldots, n$ such that $\Delta_{A k}^{(t, t+1)}(1)<\Delta_{B k}^{(t, t+1)}(1)$. We can choose a set of numbers $\left\{\beta_{i}\right\}_{i=1, \ldots, n}$ such that $\beta_{i} \searrow 0, \forall i \neq k . \Delta W$ would reduce to $\beta_{k}\left(\Delta_{A k}^{(t, t+1)}(1)-\Delta_{B k}^{(t, t+1)}(1)\right)<0$, a contradiction. QED

Proof of Proposition 7

We want to find a necessary and sufficient condition for

$$
\Delta W=\sum_{i=1}^{n} \int_{0}^{1} v_{i}(p)\left[\delta_{A i}^{(t, t+1)}(p)-\delta_{B i}^{(t, t+1)}(p)\right] d p \geq 0, \forall W \in \mathbf{W}_{14}
$$

For both conditions, note that by axiom 4 we can write: $\Delta W=\sum_{i=1}^{n} \beta_{i}\left[\Delta_{A i}^{(t, t+1)}(1)-\Delta_{B i}^{(t, t+1)}(1)\right] \geq$ 0 . Let $S_{i}=\left[\Delta_{A i}^{(t, t+1)}(1)-\Delta_{B i}^{(t, t+1)}(1)\right], \forall i=1, \ldots, n$. Since by axiom $1 \beta_{i} \geq \beta_{i+1}$ and $\beta_{i} \geq 0$ by axiom 0 , we can apply Lemma 1 . Therefore, $\Delta W=\sum_{i=1}^{n} \beta_{i} S_{i} \geq 0$ if and only if $\sum_{i=1}^{k} S_{i} \geq 0$, $\forall k=1, \ldots, n$. Hence, $\Delta W \geq 0$ if and only if $\sum_{i=1}^{k} \Delta_{A i}^{(t, t+1)}(1)-\Delta_{B i}^{(t, t+1)}(1), \forall k=1, \ldots, n$. QED

\section{Proof of Proposition 8}

We want to find a necessary and sufficient condition for

$$
\Delta W=\sum_{i=1}^{n} \int_{0}^{1} v_{i}(p)\left[\delta_{A i}^{(t, t+1)}(p)-\delta_{B i}^{(t, t+1)}(p)\right] d p \geq 0, \forall W \in \mathbf{W}_{124}
$$

For both conditions note that we can apply axiom 0,1 , and 4 and Abel's decomposition as follows: $\Delta W=\sum_{i=1}^{n}\left(\beta_{i}-\beta_{i+1}\right) \sum_{k=1}^{i} S_{k}$, where $S_{k}=\Delta_{A k}^{(t, t+1)}(1)-\Delta_{B k}^{(t, t+1)}(1)$. Let $\sum_{k=1}^{i} S_{k}=\kappa_{i}$ and $\beta_{i}-$ 
$\beta_{i+1}=\omega_{i}$, by axiom $2 \omega_{i}>\omega_{i+1}, \forall i=1, \ldots, n-1$. Applying Lemma $1, \Delta W=\sum_{i=1}^{n} \omega_{i} \kappa_{i} \geq 0$ if and only if $\sum_{i=1}^{j} \kappa_{i} \geq 0, \forall j=1, \ldots, n$. Substituting in the above expression: $\Delta W \geq 0$ if and only if $\sum_{i=1}^{j} \sum_{k=1}^{i} \Delta_{A k}^{(t, t+1)}(1) \geq \sum_{i=1}^{j} \sum_{k=1}^{i} \Delta_{B k}^{(t, t+1)}(1), \forall j=1, \ldots, n$. QED 


\section{Empirical Appendix}

Table 1A. Results of each stage of the test in Proposition 1: 2002/06 vs. 2006/10.

\begin{tabular}{|c|c|c|c|c|c|}
\hline \multirow[t]{2}{*}{$\begin{array}{c}\text { Initial } \\
\text { quantile } \\
\text { group }\end{array}$} & \multicolumn{5}{|c|}{$\begin{array}{c}\text { Quintiles of income change within } \\
\text { each initial quantile group }\end{array}$} \\
\hline & 0.2 & 0.4 & 0.6 & 0.8 & 1 \\
\hline 1 & $>$ & $>$ & $>$ & $>$ & $>$ \\
\hline 2 & $>$ & $>$ & $>$ & $>$ & $>$ \\
\hline 3 & $>$ & $>$ & $>$ & $>$ & $<$ \\
\hline 4 & $>$ & $<$ & $<$ & $<$ & $<$ \\
\hline 5 & $>$ & $>$ & $>$ & $>$ & $>$ \\
\hline 6 & $<$ & $<$ & $>$ & $<$ & $>$ \\
\hline 7 & $>$ & $>$ & $>$ & $>$ & $>$ \\
\hline 8 & $>$ & $>$ & $>$ & $>$ & $>$ \\
\hline 9 & $>$ & $>$ & $>$ & $>$ & $<$ \\
\hline 10 & $<$ & $>$ & $>$ & $>$ & $<$ \\
\hline 11 & $>$ & $>$ & $>$ & $>$ & $>$ \\
\hline 12 & $>$ & $>$ & $>$ & $>$ & $>$ \\
\hline 13 & $>$ & $>$ & $>$ & $>$ & $>$ \\
\hline 14 & $>$ & $>$ & $>$ & $>$ & $>$ \\
\hline 15 & $<$ & $>$ & $>$ & $>$ & $>$ \\
\hline 16 & $>$ & $>$ & $>$ & $>$ & $>$ \\
\hline 17 & $<$ & $<$ & $>$ & $>$ & $>$ \\
\hline 18 & $<$ & $<$ & $>$ & $>$ & $>$ \\
\hline 19 & $>$ & $>$ & $>$ & $>$ & $>$ \\
\hline 20 & $>$ & $>$ & $>$ & $>$ & $>$ \\
\hline 21 & $>$ & $<$ & $>$ & $<$ & $>$ \\
\hline 22 & $>$ & $>$ & $>$ & $>$ & $>$ \\
\hline 23 & $>$ & $>$ & $>$ & $>$ & $>$ \\
\hline 24 & $<$ & $<$ & $<$ & $<$ & $<$ \\
\hline 25 & $<$ & $<$ & $>$ & $>$ & $>$ \\
\hline
\end{tabular}

Notes: "NO" the two processes cannot be ordered;

">" the 2002/06 process dominates the 2006/10;

"<" the 2002/06 process is dominated by the $2006 / 10$.

Table 3A. Results of each stage of the test in Proposition 3: $2002 / 06$ vs. 2006/10.

\begin{tabular}{|c|c|c|c|c|c|}
\hline \multirow[t]{2}{*}{$\begin{array}{l}\text { Initial } \\
\text { quantile } \\
\text { group }\end{array}$} & \multicolumn{5}{|c|}{$\begin{array}{l}\text { Quintiles of income change within } \\
\text { each initial quantile group }\end{array}$} \\
\hline & 0.2 & 0.4 & 0.6 & 0.8 & 1 \\
\hline 1 & $>$ & $>$ & $>$ & $>$ & $>$ \\
\hline 2 & $>$ & $>$ & $>$ & $>$ & $>$ \\
\hline 3 & $>$ & $>$ & $>$ & $>$ & $>$ \\
\hline 4 & $>$ & $>$ & $>$ & $>$ & $>$ \\
\hline 5 & $>$ & $>$ & $>$ & $>$ & $>$ \\
\hline 6 & $>$ & $>$ & $>$ & $>$ & $>$ \\
\hline 7 & $>$ & $>$ & $>$ & $>$ & $>$ \\
\hline 8 & $>$ & $>$ & $>$ & $>$ & $>$ \\
\hline 9 & $>$ & $>$ & $>$ & $>$ & $>$ \\
\hline 10 & $>$ & $>$ & $>$ & $>$ & $>$ \\
\hline 11 & $>$ & $>$ & $>$ & $>$ & $>$ \\
\hline 12 & $>$ & $>$ & $>$ & $>$ & $>$ \\
\hline 13 & $>$ & $>$ & $>$ & $>$ & $>$ \\
\hline 14 & $>$ & $>$ & $>$ & $>$ & $>$ \\
\hline 15 & $>$ & $>$ & $>$ & $>$ & $>$ \\
\hline 16 & $>$ & $>$ & $>$ & $>$ & $>$ \\
\hline 17 & $>$ & $>$ & $>$ & $>$ & $>$ \\
\hline 18 & $>$ & $>$ & $>$ & $>$ & $>$ \\
\hline 19 & $>$ & $>$ & $>$ & $>$ & $>$ \\
\hline 20 & $>$ & $>$ & $>$ & $>$ & $>$ \\
\hline 21 & $>$ & $>$ & $>$ & $>$ & $>$ \\
\hline 22 & $>$ & $>$ & $>$ & $>$ & $>$ \\
\hline 23 & $>$ & $>$ & $>$ & $>$ & $>$ \\
\hline 24 & $>$ & $>$ & $>$ & $>$ & $>$ \\
\hline 25 & $>$ & $>$ & $>$ & $>$ & $>$ \\
\hline
\end{tabular}

Notes: See Notes on Table $1 \mathrm{~A}$.
Table 2A. Results at each stage of the test in Proposition 2: 2002/06 vs. 2006/10.

\begin{tabular}{|c|c|c|c|c|c|}
\hline \multirow[t]{2}{*}{$\begin{array}{l}\text { Initial } \\
\text { quantile } \\
\text { group }\end{array}$} & \multicolumn{5}{|c|}{$\begin{array}{l}\text { Quintiles of income change within } \\
\text { each initial quantile group }\end{array}$} \\
\hline & 0.2 & 0.4 & 0.6 & 0.8 & 1 \\
\hline 1 & $>$ & $>$ & $>$ & $>$ & $>$ \\
\hline 2 & $>$ & $>$ & $>$ & $>$ & $>$ \\
\hline 3 & $>$ & $>$ & $>$ & $>$ & $>$ \\
\hline 4 & $>$ & $>$ & $>$ & $>$ & $>$ \\
\hline 5 & $>$ & $>$ & $>$ & $>$ & $>$ \\
\hline 6 & $>$ & $>$ & $>$ & $>$ & $>$ \\
\hline 7 & $>$ & $>$ & $>$ & $>$ & $>$ \\
\hline 8 & $>$ & $>$ & $>$ & $>$ & $>$ \\
\hline 9 & $>$ & $>$ & $>$ & $>$ & $>$ \\
\hline 10 & $>$ & $>$ & $>$ & $>$ & $>$ \\
\hline 11 & $>$ & $>$ & $>$ & $>$ & $>$ \\
\hline 12 & $>$ & $>$ & $>$ & $>$ & $>$ \\
\hline 13 & $>$ & $>$ & $>$ & $>$ & $>$ \\
\hline 14 & $>$ & $>$ & $>$ & $>$ & $>$ \\
\hline 15 & $>$ & $>$ & $>$ & $>$ & $>$ \\
\hline 16 & $>$ & $>$ & $>$ & $>$ & $>$ \\
\hline 17 & $>$ & $>$ & $>$ & $>$ & $>$ \\
\hline 18 & $>$ & $>$ & $>$ & $>$ & $>$ \\
\hline 19 & $>$ & $>$ & $>$ & $>$ & $>$ \\
\hline 20 & $>$ & $>$ & $>$ & $>$ & $>$ \\
\hline 21 & $>$ & $>$ & $>$ & $>$ & $>$ \\
\hline 22 & $>$ & $>$ & $>$ & $>$ & $>$ \\
\hline 23 & $>$ & $>$ & $>$ & $>$ & $>$ \\
\hline 24 & $>$ & $>$ & $>$ & $>$ & $>$ \\
\hline 25 & $>$ & $>$ & $>$ & $>$ & $>$ \\
\hline
\end{tabular}

Notes: Notes: See Notes on Table $1 \mathrm{~A}$.

Table 4A. Results at each stage of the test in Proposition 4: 2002/06 vs. 2006/10

\begin{tabular}{|c|c|c|c|c|c|}
\hline \multirow{2}{*}{$\begin{array}{c}\text { Initial } \\
\text { quantile } \\
\text { group }\end{array}$} & \multicolumn{5}{|c|}{$\begin{array}{l}\text { Quintiles of income change within } \\
\text { each initial quantile group }\end{array}$} \\
\hline & 0.2 & 0.4 & 0.6 & 0.8 & 1 \\
\hline 1 & $>$ & $>$ & $>$ & $>$ & $>$ \\
\hline 2 & $>$ & $>$ & $>$ & $>$ & $>$ \\
\hline 3 & $>$ & $>$ & $>$ & $>$ & $>$ \\
\hline 4 & $>$ & $>$ & $<$ & $<$ & $<$ \\
\hline 5 & $>$ & $>$ & $>$ & $>$ & $>$ \\
\hline 6 & $<$ & $<$ & $<$ & $<$ & $<$ \\
\hline 7 & $>$ & $>$ & $>$ & $>$ & $>$ \\
\hline 8 & $>$ & $>$ & $>$ & $>$ & $>$ \\
\hline 9 & $>$ & $>$ & $>$ & $>$ & $>$ \\
\hline 10 & $<$ & $<$ & $>$ & $>$ & $<$ \\
\hline 11 & $>$ & $>$ & $>$ & $>$ & $>$ \\
\hline 12 & $>$ & $>$ & $>$ & $>$ & $>$ \\
\hline 13 & $>$ & $>$ & $>$ & $>$ & $>$ \\
\hline 14 & $>$ & $>$ & $>$ & $>$ & $>$ \\
\hline 15 & $<$ & $>$ & $>$ & $>$ & $>$ \\
\hline 16 & $>$ & $>$ & $>$ & $>$ & $>$ \\
\hline 17 & $<$ & $<$ & $>$ & $>$ & $>$ \\
\hline 18 & $<$ & $<$ & $<$ & $>$ & $>$ \\
\hline 19 & $>$ & $>$ & $>$ & $>$ & $>$ \\
\hline 20 & $>$ & $>$ & $>$ & $>$ & $>$ \\
\hline 21 & $>$ & $>$ & $>$ & $>$ & $>$ \\
\hline 22 & $>$ & $>$ & $>$ & $>$ & $>$ \\
\hline 23 & $>$ & $>$ & $>$ & $>$ & $>$ \\
\hline 24 & $<$ & $<$ & $<$ & $<$ & $<$ \\
\hline 25 & $<$ & $<$ & $<$ & $>$ & $>$ \\
\hline
\end{tabular}

Notes: See Notes on Table $1 \mathrm{~A}$. 
Table 5A. Results at each stage of the test in Proposition 5: 2002/06 vs. 2006/10.

\begin{tabular}{|c|c|c|c|c|c|}
\hline \multirow{2}{*}{$\begin{array}{c}\text { Initial } \\
\text { quantile } \\
\text { group }\end{array}$} & \multicolumn{5}{|c|}{$\begin{array}{l}\text { Quintiles of income change within } \\
\text { each initial quantile group }\end{array}$} \\
\hline & 0.2 & 0.4 & 0.6 & 0.8 & 1 \\
\hline 1 & $>$ & $>$ & $>$ & $>$ & $>$ \\
\hline 2 & $>$ & $>$ & $>$ & $>$ & $>$ \\
\hline 3 & $>$ & $>$ & $>$ & $>$ & $>$ \\
\hline 4 & $>$ & $>$ & $>$ & $>$ & $>$ \\
\hline 5 & $>$ & $>$ & $>$ & $>$ & $>$ \\
\hline 6 & $>$ & $>$ & $>$ & $>$ & $>$ \\
\hline 7 & $>$ & $>$ & $>$ & $>$ & $>$ \\
\hline 8 & $>$ & $>$ & $>$ & $>$ & $>$ \\
\hline 9 & $>$ & $>$ & $>$ & $>$ & $>$ \\
\hline 10 & $>$ & $>$ & $>$ & $>$ & $>$ \\
\hline 11 & $>$ & $>$ & $>$ & $>$ & $>$ \\
\hline 12 & $>$ & $>$ & $>$ & $>$ & $>$ \\
\hline 13 & $>$ & $>$ & $>$ & $>$ & $>$ \\
\hline 14 & $>$ & $>$ & $>$ & $>$ & $>$ \\
\hline 15 & $>$ & $>$ & $>$ & $>$ & $>$ \\
\hline 16 & $>$ & $>$ & $>$ & $>$ & $>$ \\
\hline 17 & $>$ & $>$ & $>$ & $>$ & $>$ \\
\hline 18 & $>$ & $>$ & $>$ & $>$ & $>$ \\
\hline 19 & $>$ & $>$ & $>$ & $>$ & $>$ \\
\hline 20 & $>$ & $>$ & $>$ & $>$ & $>$ \\
\hline 21 & $>$ & $>$ & $>$ & $>$ & $>$ \\
\hline 22 & $>$ & $>$ & $>$ & $>$ & $>$ \\
\hline 23 & $>$ & $>$ & $>$ & $>$ & $>$ \\
\hline 24 & $>$ & $>$ & $>$ & $>$ & $>$ \\
\hline 25 & $>$ & $>$ & $>$ & $>$ & $>$ \\
\hline
\end{tabular}

Notes: See Notes on Table 1A.

Table 7A. Results at each stage of the test in Proposition 7: 2002/06 vs. 2006/10.

\begin{tabular}{|c|c|}
\hline $\begin{array}{c}\text { Initial } \\
\text { quantile } \\
\text { group }\end{array}$ & Absolute na-gic \\
\hline 1 & $>$ \\
\hline 2 & $>$ \\
\hline 3 & $>$ \\
\hline 4 & $>$ \\
\hline 5 & $>$ \\
\hline 6 & $>$ \\
\hline 7 & $>$ \\
\hline 8 & $>$ \\
\hline 9 & $>$ \\
\hline 10 & $>$ \\
\hline 11 & $>$ \\
\hline 12 & $>$ \\
\hline 13 & $>$ \\
\hline 14 & $>$ \\
\hline 15 & $>$ \\
\hline 16 & $>$ \\
\hline 17 & $>$ \\
\hline 18 & $>$ \\
\hline 19 & $>$ \\
\hline 20 & $>$ \\
\hline 21 & $>$ \\
\hline 22 & $>$ \\
\hline 23 & $>$ \\
\hline 24 & $>$ \\
\hline 25 & $>$ \\
\hline
\end{tabular}

Notes: See Notes on Table $1 \mathrm{~A}$.
Table 6A. Results at each stage of the test in Proposition 6: 2002/06 vs. 2006/10.

\begin{tabular}{|c|c|}
\hline $\begin{array}{c}\text { Initial } \\
\text { quantile } \\
\text { group }\end{array}$ & Absolute na-gic \\
\hline 1 & $>$ \\
\hline 2 & $>$ \\
\hline 3 & $>$ \\
\hline 4 & $<$ \\
\hline 5 & $>$ \\
\hline 6 & $<$ \\
\hline 7 & $>$ \\
\hline 8 & $>$ \\
\hline 9 & $>$ \\
\hline 10 & $<$ \\
\hline 11 & $>$ \\
\hline 12 & $>$ \\
\hline 13 & $>$ \\
\hline 14 & $>$ \\
\hline 15 & $>$ \\
\hline 16 & $>$ \\
\hline 17 & $>$ \\
\hline 18 & $>$ \\
\hline 19 & $>$ \\
\hline 20 & $>$ \\
\hline 21 & $>$ \\
\hline 22 & $>$ \\
\hline 23 & $>$ \\
\hline 24 & $<$ \\
\hline 25 & $>$ \\
\hline
\end{tabular}

Notes: See Notes on Table 1A.

Table 8A. Results at each stage of the test in Proposition 8: 2002/06 vs. 2006/10.

\begin{tabular}{cl}
\hline $\begin{array}{c}\text { Initial } \\
\text { quantile } \\
\text { group }\end{array}$ & \\
& \\
1 & $>$ \\
2 & $>$ \\
3 & $>$ \\
4 & $>$ \\
5 & $>$ \\
6 & $>$ \\
7 & $>$ \\
8 & $>$ \\
9 & $>$ \\
10 & $>$ \\
11 & $>$ \\
12 & $>$ \\
13 & $>$ \\
14 & $>$ \\
15 & $>$ \\
16 & $>$ \\
17 & $>$ \\
18 & $>$ \\
19 & $>$ \\
20 & $>$ \\
21 & $>$ \\
22 & $>$ \\
23 & $>$ \\
24 & \\
25 & $>$
\end{tabular}

Notes: See Notes on Table 1A. 
Figure 1A. Non-anonymous GIC for Italy, 2002/06 vs. 2006/10.

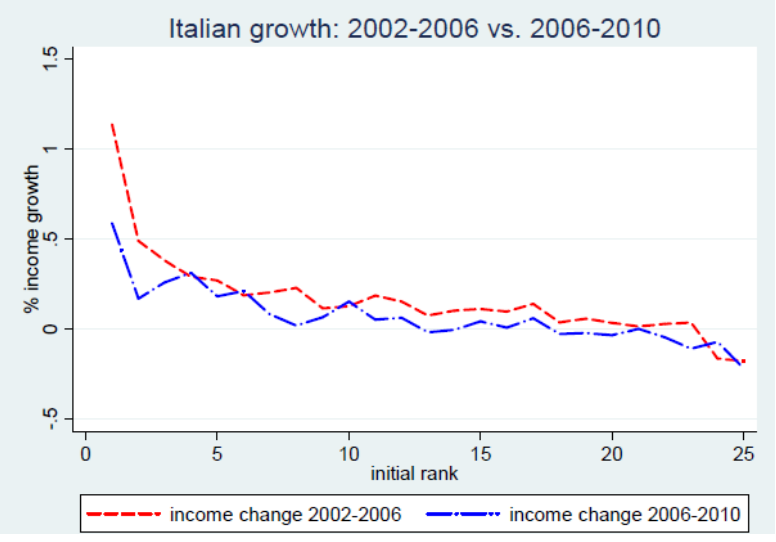

Figure 3A. Cumulative non- anonymous GIC for Italy, 2002/06 vs. 2006/10.

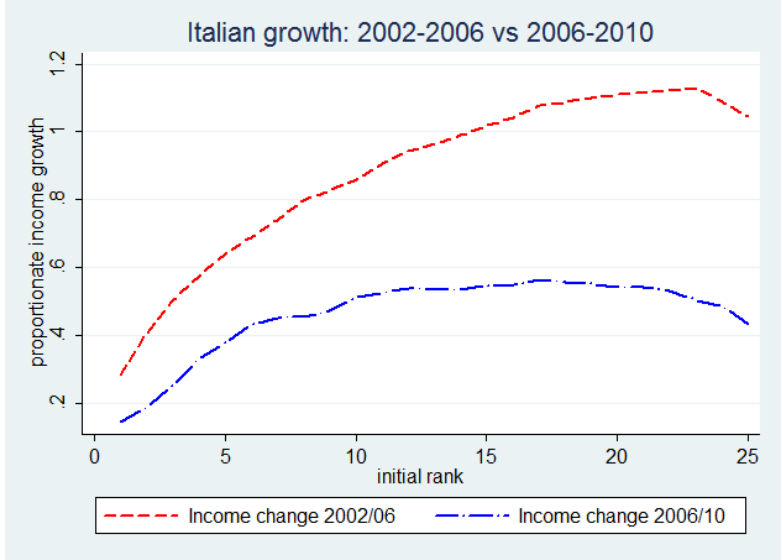

Figure 2A. GIC for Italy, 2002/06 vs. 2006/10.

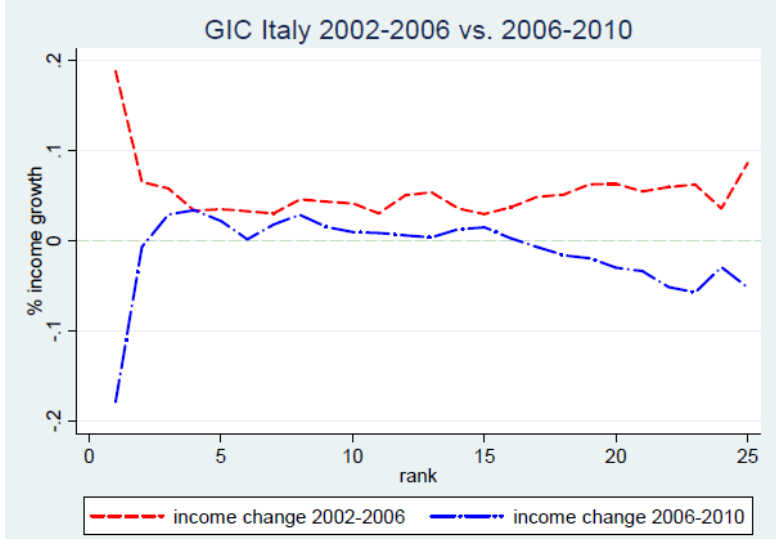

Figure 4A. Cumulative GIC for Italy, 2002/06 vs. 2006/10.

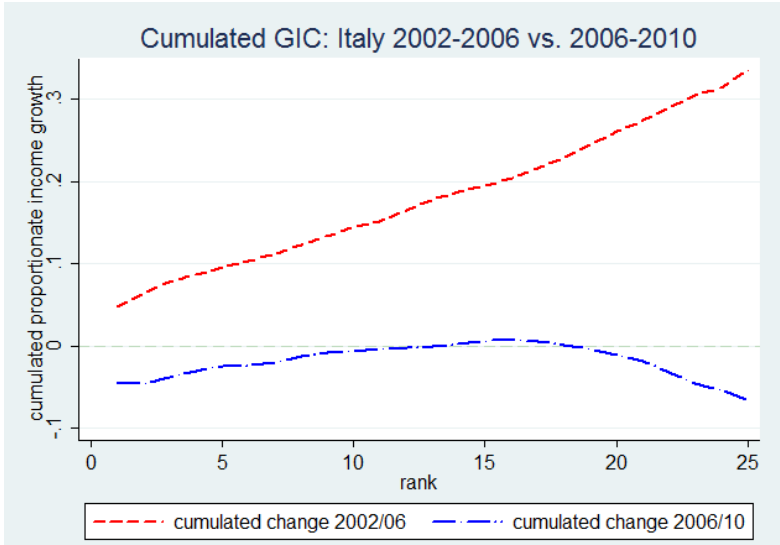

